\title{
Coordinated Stability Control Strategy for Intelligent Electric Vehicles Using Vague Set Theory
}

\author{
Yilin He $\mathbb{D}^{D}$, Jian Ma ${ }^{\mathbb{D}}$, Xuan Zhao $\mathbb{D}^{D}$, Ruoyang Song $\mathbb{D}^{\circ}$, Xiaodong Liu, and Lu Wang \\ School of Automobile, Chang'an University, Xi'an 710064, China \\ Correspondence should be addressed to Jian Ma; majian@chd.edu.cn
}

Received 9 October 2019; Revised 11 December 2019; Accepted 21 December 2019; Published 19 February 2020

Academic Editor: Jose de Jesus Rubio

Copyright (c) 2020 Yilin He et al. This is an open access article distributed under the Creative Commons Attribution License, which permits unrestricted use, distribution, and reproduction in any medium, provided the original work is properly cited.

\begin{abstract}
Aiming at improving the tracking stability performance for intelligent electric vehicles, a novel stability coordinated control strategy based on preview characteristics is proposed in this paper. Firstly, the traditional stability control target is introduced with the two degrees of freedom model, which is realized by the sliding mode control strategy. Secondly, an auxiliary control target further amending the former one with the innovation formulation of the preview characteristics is established. At last, a multiple purpose Vague set leverages the contribution of the traditional target and the auxiliary preview target in various vehicle states. The proposed coordinated control strategy is analyzed on the MATLAB/CarSim simulation platform and verified on an intelligent electric vehicle established with A\&D5435 rapid prototyping experiment platform. Simulation and experimental results indicate that the proposed control strategy based on preview characteristics can effectively improve the tracking stability performance of intelligent electric vehicles. In the double lane change simulation, the peak value of sideslip angle, yaw rate, and lateral acceleration of the vehicle is reduced by $13.2 \%, 11.4 \%$, and $8.9 \%$ compared with traditional control strategy. The average deviations between the experimental and simulation results of yaw rate, lateral acceleration, and steering wheel angle are less than $10 \%$ at different speeds, which demonstrates the consistency between the experimental and the simulation results.
\end{abstract}

\section{Introduction}

An intelligent vehicle is equipped with various sensors and electronic systems to overtake complex driving tasks from the driver, and it can be widely applied in a variety of fields. Especially, two-wheel independently driven intelligent electric vehicle is an increasingly important part of intelligent transportation systems, whose advantage is concentrated on environmental protection, safe driving, and control flexibility. It has become the research frontier in the field of vehicle engineering and the trend of the automobile industry development, which has attracted considerable interests and efforts from academia, industry, and governments [1-4].

The development and application of the intelligent electric vehicle require a variety of technologies, among which tracking stability control plays a critical role in improving safety and comfort for autonomous driving. Vehicle state parameters are monitored and controlled in the tracking process for stability guarantee. During last decades, more attention has been paid to this research topic. The carefully chosen bounds of the vehicle yaw rate and sideslip angle are designed on the basis of tire adhesion limitation so as to prevent the vehicle from spinning out of the path [5]. An electronic power-assisted steering system is studied to improve the stability of the whole vehicle from the aspect of reducing the steering system oscillation [6]. Typically, the linear quadratic regulation (LQR) and fuzzy controller can be used to minimize transient acceleration according to the curvature of the forward path while controlling the trajectory tracking error $[7,8]$. Nevertheless, these approaches are hard to manage modeling uncertainties and unknown external disturbances. To solve the problem, an actuator based on sliding mode to improve the robustness and stability is proposed [9-14]. Besides, the adaptive robust control is also a useful method to deal with the problem [15]. Moreover, model predictive control (MPC) strategy is also a popular method to deal with the parameters affecting the stable state. 
Yaw rate, lateral acceleration, and steering wheel angle can be considered as constraints in trajectory tracking control strategy to prevent the sudden change of steering angle from causing the negative impact on driving stability [16-18]. A nonlinear MPC that combines tire model with model prediction aiming at minimizing the tracking error and stabilizing the vehicle is also illustrated $[19,20]$. The aforementioned researches increase the stability margin by limiting the sideslip angle or controlling the lateral acceleration of the vehicle during trajectory tracking. However, it is still insufficient for considering the dynamic characteristics of the vehicle. There is no guarantee that the intelligent electric vehicle has good stability performance in limited conditions. Therefore, it is necessary to design a specialized stability control strategy to interfere with the vehicle state during the tracking process.

As further explored, vehicles are stabilized by combining the trajectory deviation with dynamic characteristics $[21,22]$. Furthermore, both kinematics and dynamics characteristics are considered as a multilayer controller. In the dynamics level, a decentralized predictive controller is used to optimize the front wheel angle [23]. However, these methods neglect the environment constraints and system capabilities. In order to make full use of road friction coefficient, stability is controlled in the tire force distribution layer with the hierarchical control method for trajectory tracking [24-26]. In addition, a variety of studies consider more constraints to enhance stability $[27,28]$. Based on the optimization theory, a three-layer controller making full use of adhesion of each tire to improve the stability by additional yaw moment is designed [27]. Two objective functions are established to enhance stability and mobility, respectively. Wheel driving forces are distributed under constraints including direct yaw moment requirements, road friction condition, and motor capability [28]. Although more practical conclusions can be drawn with these constraints, road condition changes are not discussed in the above researches. Aiming at dealing with the stability issue on the split road, a wavelet controller is used to improve the vehicle stability on the basis of the equal torque distribution $[29,30]$. More generally, considering additional complexity arises due to different environmental conditions, and effects have been made to improve the adaptability of control strategy in different driving conditions. Reference [31] illustrates a kind of method to construct driving conditions for electric vehicles. A slip rate control method based on the antilock braking system (ABS) and traction control system (TCS) is utilized to improve stability performance by effectively reducing the slip ratio of the wheels with different adhesion coefficient. Vehicle stability control effect is verified through the hardware-in-loop simulation and experiment [32].

Several recent researches have highlighted the potential use of environment perception system in gathering vehicle state information since the controller of a traditional vehicle cannot access sufficient information. While based on the global position system (GPS) and inertial navigation system (INS), parameters related to vehicle dynamic stability control such as position, velocity, acceleration, and yaw rate can be obtained conveniently. Based on the GPS/INS information fusion technology, a relaxation coupling observation scheme is adopted to estimate the vehicle stability state parameters, and the estimation result is robust to the change of the road coefficient and wheel parameters, which provides a reliable basis for subsequent stability control [33]. A coordinated control strategy for trajectory tracking and stability is studied by estimating the slip rate with GPS and INS [2].

In the aforementioned scenarios, impacts of the vehicle future motion on stability performance are not implicitly considered, and only common stability control problems concerned with a traditional electric vehicle are discussed. A study [34] contributes to distributing torques of four driving wheels considering the influence from a driver on the vehicle control with a preview model. However, the influence of preview on additional yaw moment to regulate the vehicle is not deeply discussed. Since additional yaw moment is also essential to stability performance, the stability control focusing on additional yaw moment optimization with preview characteristics is worthy to be studied.

In this paper, a systemic coordinated control strategy is innovatively proposed. The main contribution of this paper can be summarised as follows. (1) The structure of a system for solving the limitation of traditional stability control strategies is put forward. Considering that traditional vehicle dynamic model, like the 2 -DoF model, cannot timely handling limited conditions, the framework of a preview-based control method helping to stabilize the vehicle is designed. (2) The method to predict future motion of the vehicle is designed. For intelligent electric vehicles, their environment perception system with expanded sensing range has proven to be much helpful to vehicle controlling. Inspired by this, we put forward the method of preview to obtain the vehicle state along the certain path in advance, and we use this prediction to intervene the vehicle motion so as to improve the effect of vehicle stability control accordingly. (3) A solution for the problem of how to modify the traditional stability control through preview characteristics is proposed. Based on the Vague set theory, different modifying schemes are considered as the set of alternatives to be selected; by scoring them, we transform this problem into seeking the optimum solution to achieve the most effective control performance.

The rest of the paper is organized as follows. The control system is firstly modelled in Section 2, followed by two separate sections with traditional dynamic stability control strategy in Section 3 and preview characteristics-based control strategy in Section 4. Section 5 gives detailed introduction of the proposed coordinated control system. Simulation results and analyses are illustrated in Section 6, followed by the conclusion in Section 7 .

\section{Modeling of the Control System}

2.1. Vehicle Dynamics Modeling. This subsection builds the twelve degrees of freedom (12-DoF) dynamics model by analyzing the forces of the vehicle. There is translational 
motion of longitudinal, lateral, and vertical direction. It is also taking yaw, pitch, roll, and the rotation of 4 wheels as well as the steering of two front wheels into account, as shown in Figure 1. Neglecting the influence of road irregularity, the following assumptions are made for the modeling:

(1) Assuming that the vehicle mass is divided into sprung mass and unsprung mass

(2) Assuming that the vehicle is traveling on a flat road, i.e., the input of the road surface unevenness excitation is not considered

(3) Assuming that four wheels are independent of each other and are always in the rolling state

The sum of the rotational kinetic energy and the translational kinetic energy of the total mass is regarded as the total kinetic energy $T$. The sum of the elastic potential energy of the tires and the suspensions as well as the gravitational potential energy of the sprung mass is regarded as the total potential energy $V$. The dissipative energy of the suspensions deforming caused by the suspension shock absorbers is denoted as $E$.

According to Lagrange modeling method, we substitute the above kinetic energy, potential energy, and dissipative energy equation of the vehicle into a special form of Lagrangian equation [35] as follows:

$$
\frac{\mathrm{d}}{\mathrm{d} t}\left(\frac{\partial T}{\partial \dot{e}_{i}}\right)-\frac{\partial T}{\partial e_{i}}+\frac{\partial V}{\partial e_{i}}+\frac{\partial E}{\partial \dot{e}_{i}}=Q_{i}
$$

where $e_{i}$ and $Q_{i}$ are the generalized coordinates and the corresponding generalized forces.

The vehicle coordinate system and the tire coordinate system are depicted as $\left(X_{v}, Y_{v}, Z_{v}\right)$ and $\left(X_{w}, Y_{w}, Z_{w}\right)$. The longitudinal, lateral, and vertical dynamics equations along $X_{v}, Y_{v}$, and $Z_{v}$ axis are

$$
\left\{\begin{array}{l}
\sum F_{x}=\left(F_{x f l}+F_{x f r}\right) \cos \delta_{f}-\left(F_{y f l}+F_{y f r}\right) \sin \delta_{f} \\
\quad+F_{x r l}+F_{x r r}-F_{f}-F_{w}, \\
\sum F_{y}=\left(F_{x f l}+F_{x f r}\right) \sin \delta_{f}+\left(F_{y f l}+F_{y f r}\right) \cos \delta_{f} \\
\quad+F_{y r l}+F_{y r r}, \\
\sum F_{z}=F_{z f l}+F_{z f r}+F_{z r l}+F_{z r r}-M g,
\end{array}\right.
$$

where $\sum F_{x}, \sum F_{y}$, and $\sum F_{z}$ are the longitudinal, lateral, and vertical forces of the vehicle/N, $F_{x f l}, F_{x f r}, F_{y f l}$, and $F_{y f r}$ are the longitudinal as well as lateral forces of left and right front wheels/N, $F_{x r}, F_{x r r}, F_{y r l}$, and $F_{y r r}$ are the longitudinal as well as lateral forces of left and right rear wheels $/ \mathrm{N}, \delta_{f}$ is the front wheel steering angle/rad, $F_{f}$ is the rolling resistance force/ $\mathrm{N}$, $F_{w}$ is the aerodynamic resistance force/N, $F_{z f l}, F_{z f r}, F_{z r l}$, and $F_{z r r}$ are the vertical forces on four wheels/ $\mathrm{N}$, and $M$ is the vehicle mass $/ \mathrm{kg}$.

The roll, pitch, and yaw dynamics equations of $X_{v}, Y_{v}$, and $Z_{v}$ axis are given as

$$
\left\{\begin{array}{l}
\sum M_{x}=\frac{B}{2} \cdot\left(F_{s 1}+F_{s 3}\right)-\frac{B}{2} \cdot\left(F_{s 2}+F_{s 4}\right)-M_{s} a_{s y} z_{s m}, \\
\sum M_{y}=-a\left(F_{s 1}+F_{s 2}\right)+b\left(F_{s 3}+F_{s 4}\right)+M_{s} a_{s x} z_{s m} \\
\quad-M_{s} a_{s z} x_{s m}, \\
\sum M_{z}=a \cdot\left(F_{x f l}+F_{x f r}\right) \sin \delta_{f}+a \cdot\left(F_{y f l}+F_{y f r}\right) \cos \delta_{f} \\
\quad-\frac{B}{2} \cdot\left(F_{x f l}-F_{x f r}\right) \cos \delta_{f}+\frac{B}{2} \cdot\left(F_{y f l}-F_{y f r}\right) \sin \delta_{f} \\
\quad-\frac{B}{2} \cdot\left(F_{x r l}-F_{x r r}\right)-b \cdot\left(F_{y r l}+F_{y r r}\right)+T_{t L} \\
+T_{t R}+M_{s} a_{s y} x_{s m},
\end{array}\right.
$$

where $\sum M_{x}, \sum M_{y}$, and $\sum M_{z}$ are the rolling, pitching, and yawing moments of the vehicle/(N.m), $B$ is the wheel base/ $\mathrm{m}, a$ and $b$ are the distances from the C.G. to front and rear axles $/ \mathrm{m}, M_{s}$ is the sprung mass $/ \mathrm{kg}, a_{s x}, a_{s y}$, and $a_{s z}$ are the acceleration of the sprung mass along $X_{v}, Y_{v}$, and $Z_{v}$ axis/ $\left(\mathrm{m} / \mathrm{s}^{2}\right),\left(x_{s m}, 0, z_{s m}\right)$ are the coordinates of center of gravity for sprung mass in the vehicle coordinate system, $F_{s 1}, F_{s 2}, F_{s 3}$, and $F_{s 4}$ are the forces of four suspensions/ $\mathrm{N}$, and $T_{t L}$ and $T_{t R}$ are the left and right wheel steering torques/(N.m).

The motion of wheel is divided into two categories: one is rotational motion of four wheels around $Y_{w}$ axis and the other is steering motion of two steering wheels around $Z_{w}$ axis. The paper researches a distributed front-wheel independent drive intelligent electric vehicle, and the front wheels act as the steering wheels.

The dynamics equations of wheels rotating around $Y_{w}$ axis are

$$
\begin{aligned}
& \left\{\begin{array}{l}
M_{t f l}=T_{L}-F_{x f l} R-T_{B f l}, \\
M_{t f r}=T_{R}-F_{x f r} R-T_{B f r},
\end{array}\right. \\
& \left\{\begin{array}{l}
M_{t r l}=F_{x r l} R-T_{B r l}, \\
M_{t r r}=F_{x r r} R-T_{B r r},
\end{array}\right.
\end{aligned}
$$

where $M_{t f l}, M_{t f r}, M_{t r r}$, and $M_{t r r}$ are the torques of four wheels rotating around $Y_{w}$ axis/(N.m), $T_{B f l}, T_{B f r}, T_{B r}$, and $T_{B r r}$ are the braking torques of wheels/(N.m), $R$ is the wheel radius/ $\mathrm{m}$, and $T_{L}$ and $T_{R}$ are driving torques of the front wheels/ $(\mathrm{N} \cdot \mathrm{m})$.

The dynamics equations of front wheels rotating around $Z_{w}$ axis with the effect of the steering torques $T_{t L}$ and $T_{t R}$ as well as the resistance moments $M_{f L}$ and $M_{f R}$ are

$$
\left\{\begin{array}{l}
M_{t z l}=T_{t L}-M_{f L}, \\
M_{t z r}=T_{t R}-M_{f R},
\end{array}\right.
$$

where $M_{t z l}$ and $M_{t z r}$ are the steering torques of front wheels rotating around $Z_{w}$ axis/(N.m) and $M_{f L}$ and $M_{f R}$ are the 


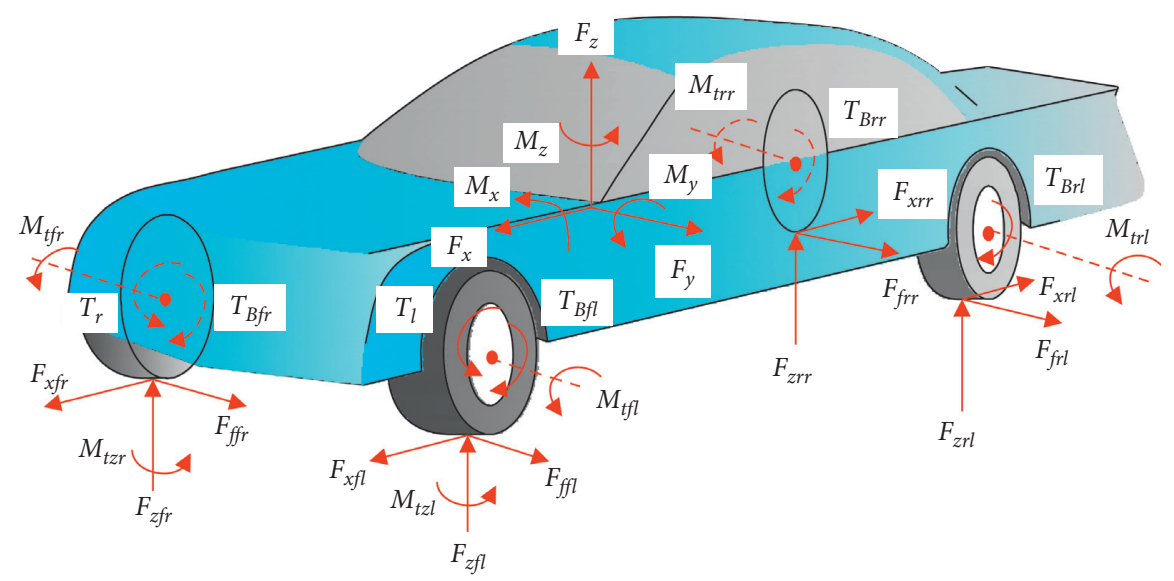

Figure 1: Vehicle dynamics model. The forces and moments of each wheel and vehicle body are demonstrated.

resistance moments of front wheels rotating around $Z_{w}$ axis/ $(\mathrm{N} \cdot \mathrm{m})$.

2.2. Tire Modeling. The longitudinal force, lateral force, and positive moment of the tire can be calculated according to the magic formula [36, 37], and certain accuracy and reliability can be ensured at the same time, which can be used to analyze the stability of the vehicle during the driving process. Therefore, magic formula is adopted to build a tire model in this paper. The typical curve of the tire model is shown in Figure 2, and the expression is shown as

$$
\begin{aligned}
Y(X)= & \left(D \operatorname { s i n } \left(C \arctan \left(B\left(X+S_{h}\right)\right)-E\left(B\left(X+S_{h}\right)\right.\right.\right. \\
& \left.\left.\left.-\arctan \left(B\left(X+S_{h}\right)\right)\right)\right)\right)+S_{v},
\end{aligned}
$$

where $Y(X)$ represents longitudinal force, lateral force, or positive moment, $X$ represents tire slip angle or slip ratio, $B$ is the stiffness factor, $C$ is the shape factor, $D$ is the peak factor, $E$ is the curvature factor, $B, C, D, E$ are the experimentally determined coefficients, $S_{h}$ is the horizontal offset/ $\mathrm{m}$, and $S_{v}$ is the vertical offset $/ \mathrm{m}$.

To simplify the calculation, the effects of $S_{h}$ and $S_{v}$ are not considered. For the intelligent electric vehicle, the front wheel steering is directly controlled by the steering motor; the lateral force of the vehicle is given by the following equation [38]:

$$
F_{y 0}=D \sin (C \arctan (B \alpha-E(B \alpha-\arctan (B \alpha)))),
$$

where $F_{y 0}$ is the lateral tire force/ $\mathrm{N}$ and $\alpha$ is the tire slip angle/ rad.

It can be known from equation (8) that the lateral tire force depends on tire slip angle between the tire's orientation and direction of travel. It is caused by the lateral elasticity of the tire, which can be described as follows:

$$
\left\{\begin{array}{l}
\alpha_{1,2}=\delta_{f}-\frac{v+a \omega_{r}}{u} \\
\alpha_{3,4}=-\frac{v-b \omega_{r}}{u}
\end{array}\right.
$$

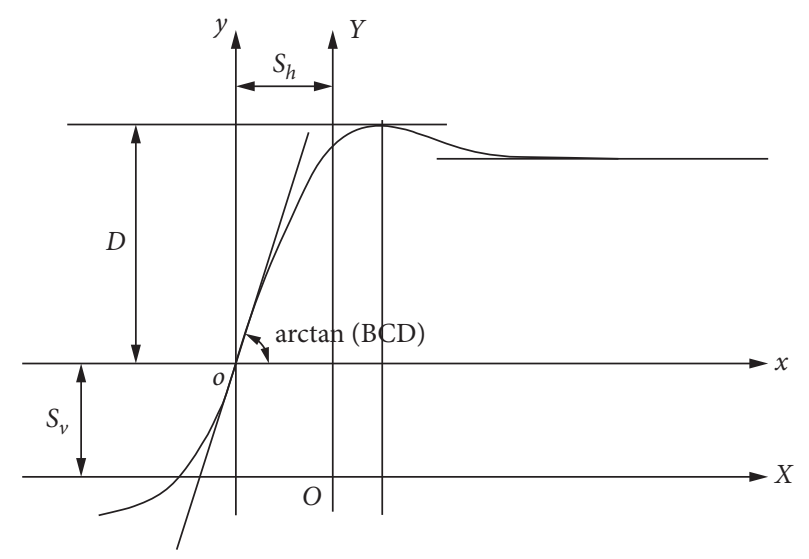

FIgURE 2: Typical curve of the tire model. Tire longitudinal force/ lateral force/positive moment varies with slip angle or slip ratio.

where $\alpha_{1,2}$ and $\alpha_{3,4}$ are the front and rear wheel slip angle/ $\mathrm{rad}, v$ is the vehicle lateral speed $/(\mathrm{km} / \mathrm{h})$, and $\omega_{r}$ is the vehicle yaw $\mathrm{rate} /(\mathrm{rad} / \mathrm{s})$.

2.3. Preview Modeling. As for a traditional vehicle, a virtual temporary target point is set on the target path by the driver, and the vehicle is controlled with the target point according to driving experience to minimize the deviation between the current position and the target position in real time. Inspired by the driving law, a single point preview model is established to track the desired trajectory by taking the vehicle's lateral deviation and heading angle deviation as the state parameters.

Taking the front axle center as the motion reference point, the point $P$ at the preview distance along the longitudinal direction of the vehicle is used as the preview point, and the mirror point $P^{\prime}$ with the same distance on the trajectory is the target point. The distance between $P$ point and $P^{\prime}$ point is the lateral deviation. The angle between the current longitudinal velocity and the tangent line of the road at $P$ point is regarded as heading angle deviation. The single point preview model is shown in Figure 3 and can be written as [39]: 


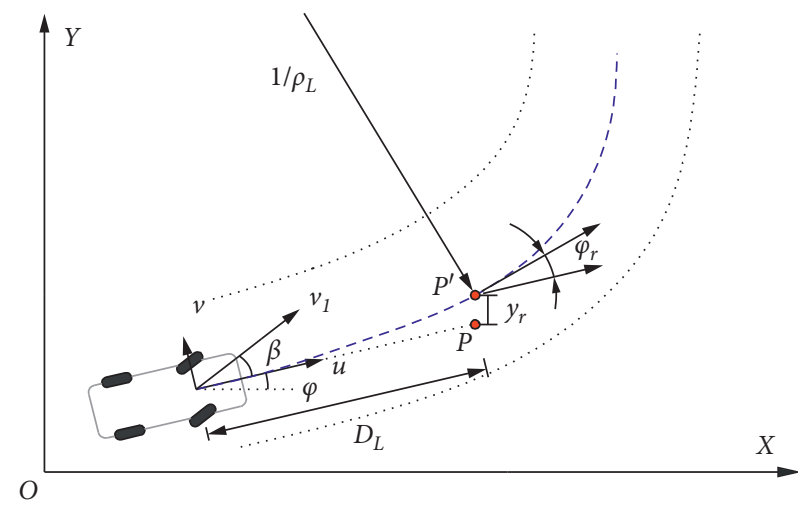

FIgURE 3: Single point preview model. The relationships between lateral deviation as well as heading angle deviation and road geometry can be seen.

$$
\left\{\begin{array}{l}
\dot{y}_{r}=u \varphi_{r}-u \beta-\dot{\varphi} D_{L}, \\
\dot{\varphi}_{r}=u \rho_{L}-\dot{\varphi},
\end{array}\right.
$$

where $D_{L}$ is the preview distance/m, $y_{r}$ is the lateral deviation $/ \mathrm{m}, \varphi_{r}$ is the heading angle deviation/rad, $u$ is the longitudinal velocity of the vehicle $/(\mathrm{km} / \mathrm{h}), \beta$ is the vehicle sideslip angle/rad, $\varphi$ is the yaw angle/rad, and $\rho_{L}$ is the curvature of the path to be followed $/ \mathrm{m}$.

The preview distance $D_{L}$ is related to vehicle speed as follows [40]:

$$
D_{L}= \begin{cases}D_{L \min }, & u \leq u_{\min } \\ k_{L} \cdot u+d, & u_{\min }<u<u_{\max } \\ D_{L \max }, & u \geq u_{\max }\end{cases}
$$

where $D_{L \min }$ is the minimum preview distance $/ \mathrm{m}, u_{\min }$ is the corresponding speed of $D_{L \min } /(\mathrm{km} / \mathrm{h}), D_{L \max }$ is the maximum preview distance limited with the maximum visual distance of the vision system $/ \mathrm{m}, u_{\max }$ is the corresponding speed of $D_{L \max } /(\mathrm{km} / \mathrm{h})$, and $k_{L}$ is the preview time $/ \mathrm{s}$, which is generally set within $[0.58,2.072]$ according to the driver's preview time range. $d$ is the fitting coefficient, which is set according to the characteristics and control requirements of the intelligent electric vehicle. In this model, $D_{L \min }, D_{L \max }$, $u_{\min }, u_{\max }, k_{L}$, and $d$ are set to $8.56 \mathrm{~m}, 36.33 \mathrm{~m}, 10 \mathrm{~km} / \mathrm{h}$, $60 \mathrm{~km} / \mathrm{h}, 2 \mathrm{~s}$, and 0.556 , respectively.

\section{Traditional Stability Control of Intelligent Electric Vehicles}

In this paper, the stability control strategy without considering preview characteristics is called the traditional stability control strategy. Considering the sliding mode is not sensitive to system parameters perturbation and external disturbance, sliding mode control (SMC) has high potential in vehicle control systems and is employed for traditional dynamic stability control in this paper. In order to guarantee vehicle sideslip angle and yaw rate can track the desired value, the structure of traditional dynamic control strategy based on the sliding mode is designed as Figure 4. The 2-DoF vehicle model acts as the desired model. Yaw rate $\omega_{r d_{1}}$ and sideslip angle $\beta_{d_{1}}$ of the vehicle based on the 2-DoF model are shown as follows $[38,41,42]$ :

$$
\begin{aligned}
& \omega_{r_{1}}^{*}=\min \left\{\left|\frac{u \delta_{f}}{L\left(1+K u^{2}\right)}\right|,\left|\frac{\mu g}{u}\right|\right\} \cdot \operatorname{sgn}\left(\delta_{f}\right), \\
& \beta_{d_{1}}^{*}=\min \left\{\left|\frac{b+\left(m a u^{2} / k_{2} L\right)}{L\left(1+K u^{2}\right)} \delta_{f}\right|,\left|\mu g\left(\frac{b}{u^{2}}+\frac{m a}{k_{2} L}\right)\right|\right\} \cdot \operatorname{sgn}\left(\delta_{f}\right) .
\end{aligned}
$$

The sliding surface based on the above control objectives can be designed, as shown in

$$
s=e_{1}+\gamma e_{2}
$$

where $e_{1}=\omega_{r}-\omega_{r d_{1}}, e_{2}=\beta-\beta_{d_{1}}$, and $\gamma$ is the weight coefficient, which represents the proportion of the sideslip angle deviation in the total value [43].

Through the 12-DoF vehicle model established in the previous section, the following equation can be obtained:

$$
\begin{aligned}
\dot{\omega}_{r}= & \frac{1}{I_{z}}\left[a \cdot\left(F_{x f l}+F_{x f r}\right) \sin \delta_{f}+a \cdot\left(F_{y f l}+F_{y f r}\right) \cos \delta_{f}\right. \\
& -\frac{B}{2} \cdot\left(F_{x f l}-F_{x f r}\right) \cos \delta_{f}+\frac{B}{2} \cdot\left(F_{y f l}-F_{y f r}\right) \sin \delta_{f} \\
& -\frac{B}{2} \cdot\left(F_{x r l}-F_{x r r}\right)-b \cdot\left(F_{y r l}+F_{y r r}\right)+T_{t L}+T_{t R} \\
& \left.+M_{s} a_{s y} x_{s m}\right] .
\end{aligned}
$$

The yaw moment generated by the driving torque can be depicted as

$$
M=a \cdot\left(F_{x f l}+F_{x f r}\right) \sin \delta_{f}-\frac{B}{2} \cdot\left(F_{x f l}-F_{x f r}\right) \cos \delta_{f} .
$$

The derivative of $s$ can be calculated as

$$
\begin{aligned}
\dot{s}= & \frac{1}{I_{z}}\left[a \cdot\left(F_{y f l}+F_{y f r}\right) \cos \delta_{f}+\frac{B}{2} \cdot\left(F_{y f l}-F_{y f r}\right) \sin \delta_{f}\right. \\
& -b \cdot\left(F_{y r l}+F_{y r r}\right) \\
& \left.-\frac{B}{2} \cdot\left(F_{x r l}-F_{x r r}\right)+T_{t L}+T_{t R}+M_{s} a_{s y} x_{s m}+M+U\right],
\end{aligned}
$$

where $U=I_{z}\left[\gamma\left(\dot{\beta}-\dot{\beta}_{d_{1}}^{*}\right)-\dot{\omega}_{r d_{1}}^{*}\right] . U$ in equation (16) is also bounded, $|U|<\widetilde{U}$, with $\widetilde{U}$ being the boundary.

Setting $\dot{s}=0$, equivalent control law can be obtained:

$$
\begin{aligned}
u_{\mathrm{eq}}= & -a \cdot\left(F_{y f l}+F_{y f r}\right) \cos \delta_{f}-\frac{B}{2} \cdot\left(F_{y f l}-F_{y f r}\right) \sin \delta_{f} \\
& +b \cdot\left(F_{y r l}-F_{y r r}\right)+\frac{B}{2} \cdot\left(F_{x r l}-F_{x r r}\right)-T_{t L}-T_{t R} \\
& -M_{s} a_{s y} x_{s m} .
\end{aligned}
$$




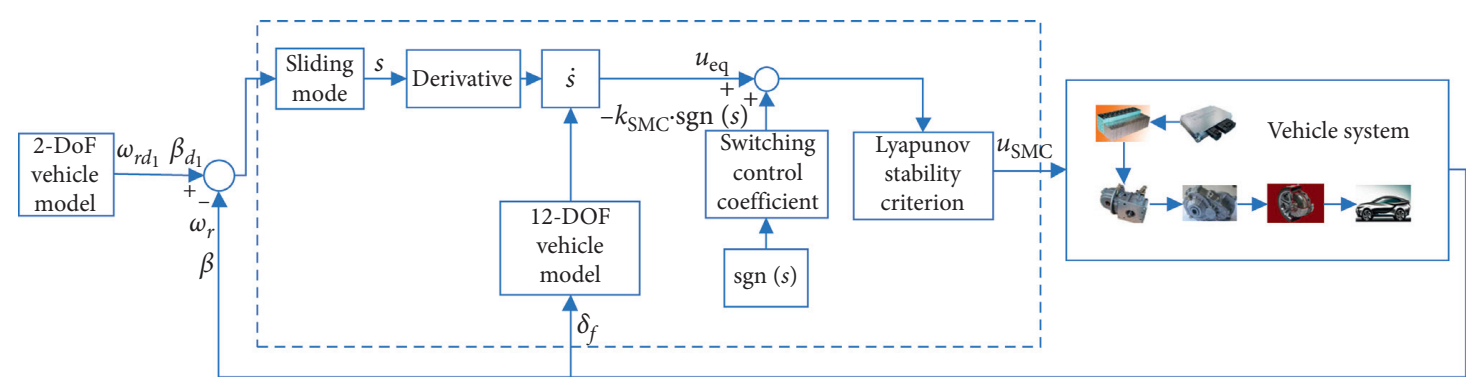

FIgURE 4: Structure of traditional stability control. The ideal vehicle state is calculated with 2-DoF vehicle model, and the required control output of SMC.

Considering the real-time requirements in controlling process, constant rate approaching method is selected to design the controller to improve response speed [44] as follows:

$$
u_{\mathrm{SMC}}=u_{\mathrm{eq}}-k_{\mathrm{SMC}} \cdot \operatorname{sgn}(s),
$$

where $\operatorname{sgn}(\cdot)$ is a sign function, $k_{\mathrm{SMC}}$ is the coefficient, and $k_{\text {SMC }}$ is larger than $\widetilde{U}$.

In order to verify the system stability, the second stability criterion of Lyapunov is adopted. Lyapunov function can be defined as

$$
V=\frac{1}{2} s^{2}
$$

The reachability condition $\dot{V}<0$ must be guaranteed when sliding.

Substituting the control law of the designed controller of equation (18) into (16)

$$
\dot{s}=\frac{1}{I_{z}}\left(U-k_{\mathrm{SMC}} \operatorname{sgn}(s)\right) .
$$
into it

Deriving equation (19) and substituting equation (20)

$$
\dot{V}=\frac{U s-k_{\mathrm{SMC}} \operatorname{sgn}(s) s}{I_{z}}<\frac{\left(\widetilde{U}-k_{\mathrm{SMC}}\right)|s|}{I_{z}} .
$$

Since $k_{S M C}>\widetilde{U}$ can be guaranteed during the sliding mode controller designing process, it is obvious that $\dot{V}<0$ can be satisfied.

\section{Tracking Stability Control of Intelligent Electric Vehicles Based on Preview Characteristics}

4.1. Tracking Stability Control Target. Since the driving condition is complex and changeable, the vehicle dynamics characteristics are inclined to change during the trajectory tracking process. Besides, the simplified 2-DoF model cannot accurately describe the controlled system at any time. Figure 5 gives the relationship between desired yaw rate and vehicle velocity under various road friction coefficients $\mu$ and steering angles $\delta$.

Assuming when vehicle drives on the road with the friction coefficient of 0.5 and the steering angle is $7^{\circ}$, with the increasing of vehicle speed, the yaw rate also increases. At

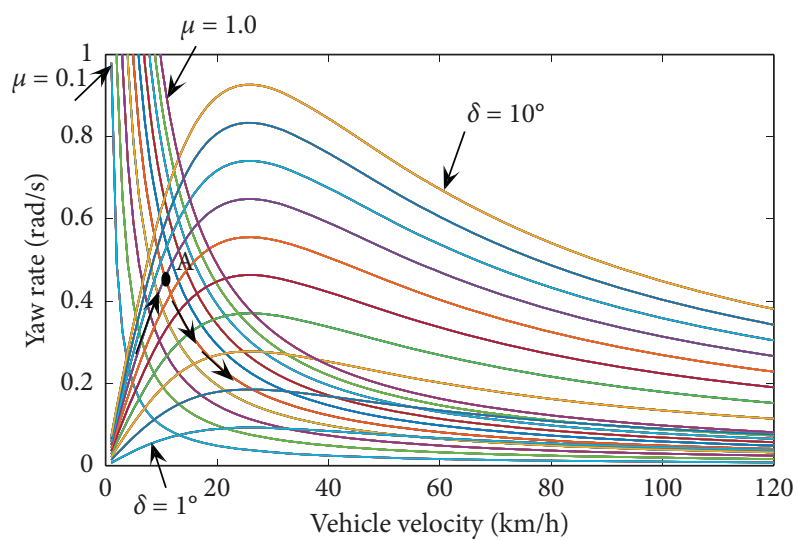

FIgure 5: The desired yaw rate with respect to vehicle velocity at different friction coefficient roads from 0.1 to 1.0 and steering wheel angles from $1^{\circ}$ to $10^{\circ}$ is demonstrated.

point $\mathrm{A}$, with the restriction of friction coefficient, yaw rate begins to decrease. The deviation between ideal value and actual value is smaller than the deviation required for actual stability control. As a result, the feedback control output also decreases. Therefore, the stability control of yaw moment based on the 2-DoF model has certain limitations and it is hard to obtain the optimal control effect at any time, especially in extreme conditions. What is more, if the curvature of the trajectory to be tracked is fairly large, rapid and accurate response of vehicle state are expected to prevent excessive output of the front wheel steering angle and its changing rate caused by trajectory deviation with trajectory tracking control strategy, which has an unfavorable effect on stability. On the other hand, the rapid response also serves the next stage of preview [45].

Generally, a driver's handling behavior will also exert an important impact on the stability in the course of driving for the traditional vehicle. The state of the vehicle can be adjusted according to the driver's subjective feelings [46, 47]. The driving process of an intelligent electric vehicle, however, lacks subjective intervention of a driver, which will impose a negative impact on vehicle stability to a certain extent. Therefore, a trajectory tracking stability control strategy based on the preview characteristics is proposed. With the preview model established in Section 2, the future movement of the vehicle can be predicted by observing the destination path in advance and the parameters related to vehicle stability state can be obtained. As a result, driving 
behavior of the intelligent electric vehicle equivalent to driver's intention in a traditional vehicle can be obtained, through which the direct yaw moment of the stability control determined with the 2-DoF model can be optimized. According to the vehicle preview model in equation (10), the desired vehicle sideslip angle and yaw rate satisfying the lateral deviation and heading angle deviation can be written as follows:

$$
\left\{\begin{array}{l}
\omega_{r d_{2}}^{*}=u \cdot \rho_{L}-\dot{\varphi}_{r}, \\
\beta_{d_{2}}^{*}=\frac{u \varphi_{r}-\dot{y}_{r}-\left(u \cdot \rho_{L}-\dot{\varphi}_{r}\right) \cdot D_{L}}{u} .
\end{array}\right.
$$

4.2. LQR Tracking Stability Control Strategy. LQR is adopted in tracking stability control strategy in this paper with regard to its advantage in forming a closed loop optimal control. According to LQR control theory, state variables are the deviations between actual yaw rate as well as sideslip angle and the ideal values based on the trajectory tracking stability control target during the preview and follow process. The yaw moment $M_{2}$ acting on the intelligent electric vehicle is the control input. To sum up, the tracking stability model can be expressed in a continuous form as follows [48]:

$$
\left[\begin{array}{c}
\Delta \dot{\beta} \\
\Delta \dot{\omega}_{r}
\end{array}\right]=\mathbf{A}_{2} \cdot\left[\begin{array}{c}
\Delta \beta \\
\Delta \omega_{r}
\end{array}\right]+\mathbf{B}_{2} \cdot M_{2}
$$

where $\quad \mathbf{A}_{2}=\left[\begin{array}{cc}\left(b k_{2}-a k_{1} / I_{z}\right) & -\left(a^{2} k_{1}+b^{2} k_{2} / I_{z} u\right) \\ \left(-\left(k_{1}+k_{2} / m u\right)\right) & \left(b k_{2}-a k_{1} / m u\right)-u\end{array}\right]$, $\mathbf{B}_{2}=\left[-\left(1 / I_{z}\right), 0\right]^{\mathrm{T}}$, and $I_{z}$ is the vehicle's moment of inertia.

The evaluation index of the system is shown as

$$
J_{2}=0.5 \int_{0}^{\infty}\left[\mathbf{x}^{\mathrm{T}}(t) \mathbf{Q}_{2}(t) \mathbf{x}(t)+\mathbf{u}^{\mathrm{T}}(t) \mathbf{R}_{2}(t) \mathbf{u}(t)\right] \mathrm{d} t,
$$

where the error integral term $\left(0.5 \mathbf{x}^{\mathrm{T}}(t) \mathbf{Q}_{2}(t) \mathbf{x}(t)\right)$ denotes the sum of system state error, which represents the total measurement for the tracking errors of the sideslip angle and yaw rate. When $\mathbf{x}(t) \neq 0, \mathbf{x}^{\mathrm{T}}(t) \mathbf{Q}_{2}(t) \mathbf{x}(t) \geq 0$, the value of the item is proportional to the error, which reflects the control effect of the system. The control integral term $\left(0.5 \mathbf{u}^{\mathrm{T}}(t) \mathbf{R}_{2}(t) \mathbf{u}(t)\right)$ represents the measurement of yaw moment generated in the stability control process of trajectory tracking. The value of this item is proportional to the energy consumed, which reflects the cost of control. $\mathbf{Q}_{2}$ and $\mathbf{R}_{2}$ are the weight matrix for control accuracy and energy cost during the control, respectively. $\mathbf{Q}_{2}$ is set to a diagonal matrix, $\mathbf{Q}_{2}=\left[\begin{array}{cc}q_{1} & 0 \\ 0 & q_{2}\end{array}\right]$. The structure of the preview stability control is shown in Figure 6.

Control vector can be written as

$$
u_{L}(t)=-\mathbf{K}_{L} \mathbf{x}(t)
$$

where $\mathbf{K}_{L}$ is a coefficient matrix.

Based on the Lyapunov function, the output of the system is continuously transmitted to the desired state. Assuming that

$$
\mathbf{x}^{\mathrm{T}}(t)\left(\mathbf{Q}_{2}+\mathbf{K}_{L}^{\mathrm{T}} \mathbf{R}_{2} \mathbf{K}_{L}\right) \mathbf{x}(t)=-\frac{\mathrm{d}}{\mathrm{d} t}\left(\mathbf{x}^{\mathrm{T}}(t) \mathbf{P} \mathbf{x}(t)\right),
$$

the degenerate Riccati equation is obtained as

$$
\left(\mathbf{A}_{2}-\mathbf{B}_{2} \mathbf{K}_{L}\right)^{\mathrm{T}} \mathbf{P}+\mathbf{P}\left(\mathbf{A}_{2}-\mathbf{B}_{2} \mathbf{K}_{L}\right)=-\left(\mathbf{Q}_{2}+\mathbf{K}_{L}^{\mathrm{T}} \mathbf{R}_{2} \mathbf{K}_{L}\right) .
$$

According to Lyapunov's second method, if $\mathbf{A}_{2}-\mathbf{B}_{2} \mathbf{K}_{L}$ is a stable matrix, there exists $\mathbf{P}$ satisfying equation (27), and when $\mathbf{x}^{\mathrm{T}}(\infty)=0$, the performance index is

$$
J=\mathbf{x}^{\mathrm{T}}(0) \mathbf{P} \mathbf{x}(0) \text {. }
$$

Since $\mathbf{R}_{2}$ is a positive definite symmetric matrix, $\mathbf{R}_{2}$ can be written with a nonsingular matrix $\mathbf{T}$ as $\mathbf{R}_{2}=\mathbf{T}^{\mathrm{T}} \mathbf{T}$. Equation (27) is equivalent to

$$
\left(\mathbf{A}_{2}^{\mathrm{T}}-\mathbf{K}_{L}^{\mathrm{T}} \mathbf{B}_{2}^{\mathrm{T}}\right) \mathbf{P}+\mathbf{P}\left(\mathbf{A}_{2}-\mathbf{B}_{2} \mathbf{K}_{L}\right)+\mathbf{Q}_{2}+\mathbf{K}_{L}^{\mathrm{T}} \mathbf{T}^{\mathrm{T}} \mathbf{T} \mathbf{K}_{L}=0 .
$$

Equation (29) can be rewritten as

$$
\begin{aligned}
\mathbf{A}_{2}^{\mathrm{T}} \mathbf{P} & +\mathbf{P A}_{2}+\left[\mathbf{T} \mathbf{K}_{L}-\left(\mathbf{T}^{\mathrm{T}}\right)^{-1} \mathbf{B}_{2}^{\mathrm{T}} \mathbf{P}\right]^{\mathrm{T}}\left[\mathbf{T} \mathbf{K}_{L}-\left(\mathbf{T}^{\mathrm{T}}\right)^{-1} \mathbf{B}_{2}^{\mathrm{T}} \mathbf{P}\right] \\
& -\mathbf{P B}_{2} \mathbf{R}_{2}^{-1} \mathbf{B}_{2}^{\mathrm{T}} \mathbf{P}+\mathbf{Q}_{2}=0
\end{aligned}
$$

In order to minimize the performance index, $\mathbf{K}_{L}$ is minimized by

$$
\mathbf{x}^{\mathrm{T}}(t)\left[\mathbf{T K}_{L}-\left(\mathbf{T}^{\mathrm{T}}\right)^{-1} \mathbf{B}_{2}^{\mathrm{T}} \mathbf{P}\right]^{\mathrm{T}}\left[\mathbf{T K}_{L}-\left(\mathbf{T}^{\mathrm{T}}\right)^{-1} \mathbf{B}_{2}^{\mathrm{T}} \mathbf{P}\right] \mathbf{x}(t)=0 .
$$

Then,

$$
\mathbf{K}_{L}=\mathbf{T}^{-1}\left(\mathbf{T}^{\mathrm{T}}\right)^{-1} \mathbf{B}_{2}^{\mathrm{T}} \mathbf{P}=\mathbf{R}_{2}^{-1} \mathbf{B}_{2}^{\mathrm{T}} \mathbf{P},
$$

where $\mathbf{R}_{2}$ is a constant matrix.

Equation (32) must satisfy the degenerate Riccati equation, and the control vector can be obtained by submitting $\mathbf{K}_{L}$ into equation (25).

\section{Coordinated Tracking Stability Control Strategy of Intelligent Electric Vehicles}

5.1. Overall Scheme of Coordinated Tracking Stability Control. The proposed coordinated stability control strategy leverages two control objectives from both traditional dynamic stability control and preview characteristics-based control. The former control method enables the vehicle to track the desired sideslip angle and yaw rate of the 2-DoF model, while the latter method can effectively reflect the driving behavior of the vehicle so as to be adopted to amend the former control.

The correction factor $\varepsilon$ and $1-\varepsilon$ are introduced to adaptively define the relative contributions of traditional dynamic stability control target and the previewing stability control target. The final yaw moment is formulated as

$$
M_{z}=\varepsilon M_{1}+(1-\varepsilon) M_{2} \text {, }
$$




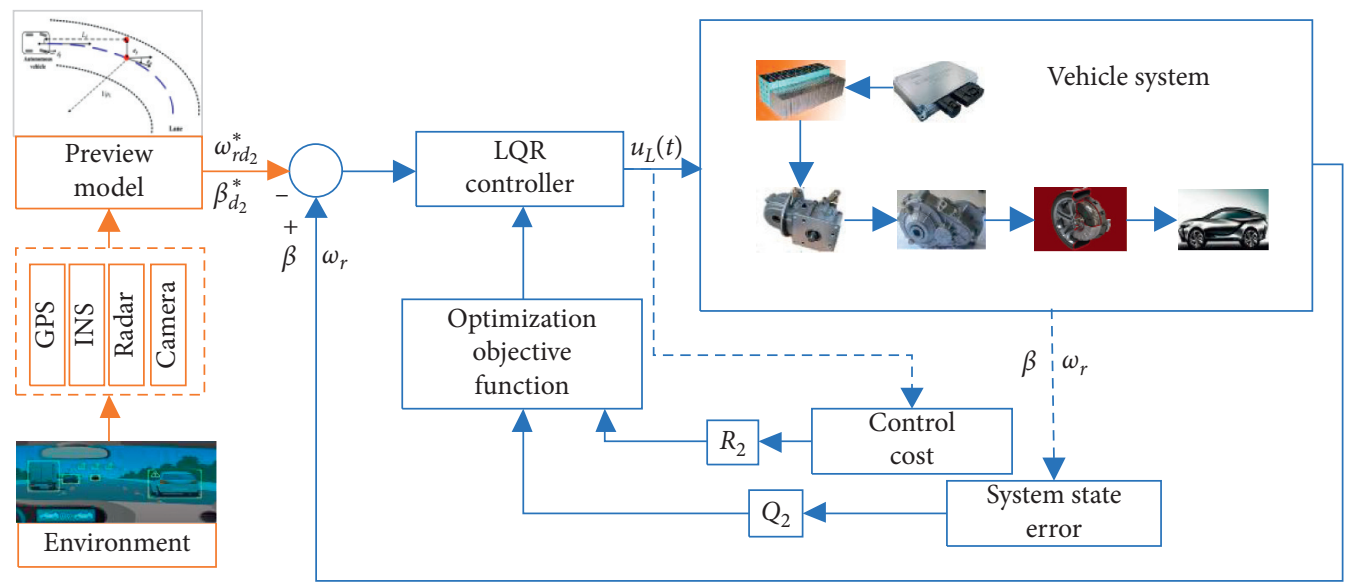

FIGURE 6: Structure of the preview stability control. The ideal vehicle state is calculated with preview model, and the required control output of LQR acts on vehicle system.

where $M_{1}$ is the traditional dynamic stability control part, $M_{1}=u_{\mathrm{SMC}}=u_{\mathrm{eq}}-k_{\mathrm{SMC}} \cdot \operatorname{sat}(s) ; M_{2}$ is the part reflecting preview characteristics, $M_{2}=-\mathbf{K}_{L} \cdot \mathbf{x}(t)=-k_{\beta} \cdot \Delta \beta(t)-$ $k_{\omega_{r}} \cdot \Delta \omega_{r}(t)$. The calculations of $M_{1}$ and $M_{2}$ are shown in Sections 3 and 4.2, respectively.

It can be seen that the chosen correction factor will affect the final stability control performance. However, due to the fuzziness and uncertainty of information, it is difficult for traditional methods to integrate two control objectives directly. While multiobjective decision with Vague set has obvious advantages in dealing with the uncertainty of multiple control objectives and is adopted to calculate the correction factor [49]. The structure of the proposed coordinated control strategy can be depicted in Figure 7 . Vehicle state and control outputs of the two different targets are regarded as Vague set input.

\subsection{Design of Tracking Stability Coordinated Control Strategy} with Multiple Purpose Vague Set. A scheme set $A^{*}$ is the candidate set of control methods. In this paper, $A_{1}^{*}$ represents the traditional stability control strategy, $A_{2}^{*}$ is the preview stability control strategy, and $A_{3}^{*}$ is the coordinated control scheme, where both contributions of former objectives are combined. Therefore, the scheme set can be written as $A^{*}=\left\{A_{1}^{*}, A_{2}^{*}, A_{3}^{*}\right\}$. $C_{1}$ is the constraint with 2-DoF model; $C_{2}$ is the constraint with preview model, so the constraint condition is $C=\left\{C_{1}, C_{2},\right\} . A_{i}^{*}$ in $C$ is characterized by the following Vague set:

$$
A_{i}^{*}=\left\{\left(C_{1},\left[t_{i 1}, 1-f_{i 1}\right]\right),\left(C_{2},\left[t_{i 2}, 1-f_{i 2}\right]\right)\right\},
$$

where $t_{i j}$ denotes the grade that $A_{i}^{*}$ satisfies $C_{j}$, while $f_{i j}$ denotes the grade that $A_{i}^{*}$ does not satisfy $C_{j}, j=1,2$.

According to the characteristics of different stability control schemes, the Vague set is defined as

$$
\begin{cases}A_{1}^{*}=\left\{C_{1}\left[t_{11}, 1-f_{11}\right],\right. & \left.C_{2}\left[t_{12}, 1-f_{12}\right]\right\}, \\ A_{2}^{*}=\left\{C_{1}\left[t_{21}, 1-f_{21}\right],\right. & \left.C_{2}\left[t_{22}, 1-f_{22}\right]\right\}, \\ A_{3}^{*}=\left\{C_{1}\left[t_{31}, 1-f_{31}\right],\right. & \left.C_{2}\left[t_{32}, 1-f_{32}\right]\right\},\end{cases}
$$

where $t_{3 j}=\varepsilon t_{1 j}+(1-\varepsilon) t_{2 j}$ and $f_{3 j}=\varepsilon f_{1 j}+(1-\varepsilon) f_{2 j}$.
In order to select a control method in scheme $A^{*}$, which can satisfy both control constraints $C_{1}$ and $C_{2}$, the evaluation function $E\left(A_{i}^{*}\right)$ is designed to indicate the grade of satisfaction of method $A_{i}^{*}$ to constraints. The evaluation function is shown in

$$
\begin{aligned}
E\left(A_{i}^{*}\right) & =\left(\left[t_{i 1}, 1-f_{i 1}\right] \wedge\left[t_{i 2}, 1-f_{i 2}\right]\right) \\
& =\left[t_{A_{i}^{*}}, 1-f_{A_{i}^{*}}\right],
\end{aligned}
$$

where $t_{A_{i}^{*}}=\min \left(t_{i 1}, t_{i 2}\right)$ and $1-f_{A_{i}^{*}}=\min \left(1-f_{i 1}, 1-f_{i 2}\right)$.

We set $c_{A_{*}^{*}}=1-t_{A^{*}}-f_{A^{*}} ; t_{A_{*}^{*}}, f_{A^{*}}$, and $c_{A_{*}^{*}}$ represent the true membership, false membership, and ambiguity, respectively. Since the ambiguity may contain the parts that tend to belong to or do not belong to the two distinct attributes, the proportion of these two parts is $t_{A_{i}^{*}} c_{A_{i}^{*}}$ and $f_{A_{i}^{*}} c_{A_{i}^{*}}$. The remaining part is $\left(1-t_{A_{i}^{*}}-f_{A_{i}^{*}}\right) \cdot{ }^{{ }_{i}} c_{A_{i}^{*}}$. The score function is defined as follows:

$$
L\left(E\left(A_{i}^{*}\right)\right)=t_{A_{i}^{*}}+t_{A_{i}^{*}}\left(1-t_{A_{i}^{*}}-f_{A_{i}^{*}}\right) .
$$

In order to achieve the designed goal, the stability control effect based on Vague set should be better than that based on traditional dynamic stability control or preview stability control alone.

$$
L\left(E\left(A_{3}^{*}\right)\right)>\max \left\{L\left(E\left(A_{1}^{*}\right)\right), L\left(E\left(A_{2}^{*}\right)\right)\right\} .
$$

By solving equation (38), we can get the range of $\varepsilon$ is $\left[\varepsilon_{\min }, \varepsilon_{\max }\right]$. In this paper, the values of $t_{11}, t_{12}, f_{11}, f_{12}, t_{21}, t_{22}$, $f_{21}$, and $f_{22}$ are set to $0.9,0.1,0.1,0.8,0.9,0.2,0.1$, and 0.7 .

Considering the steering wheel angle is proportional to the degree of lateral motion for vehicle, when the steering wheel is large, priority is given to the preview stability control with increased weight so that the desired trajectory can be tracked reliably. While the steering wheel angle is small, priority should be given to ensuring that the vehicle tracks the desired state with the 2-DoF model and the weight of the preview stability control should decrease. Therefore, the correction factor $\varepsilon$ can be adjusted according to

$$
\varepsilon=\varepsilon_{\min }+\left(\varepsilon_{\max }-\varepsilon_{\min }\right) \cdot \frac{\delta_{\max }-\delta_{f}}{\delta_{\max }},
$$

where $\delta_{\max }$ is the maximum steering wheel angle. 


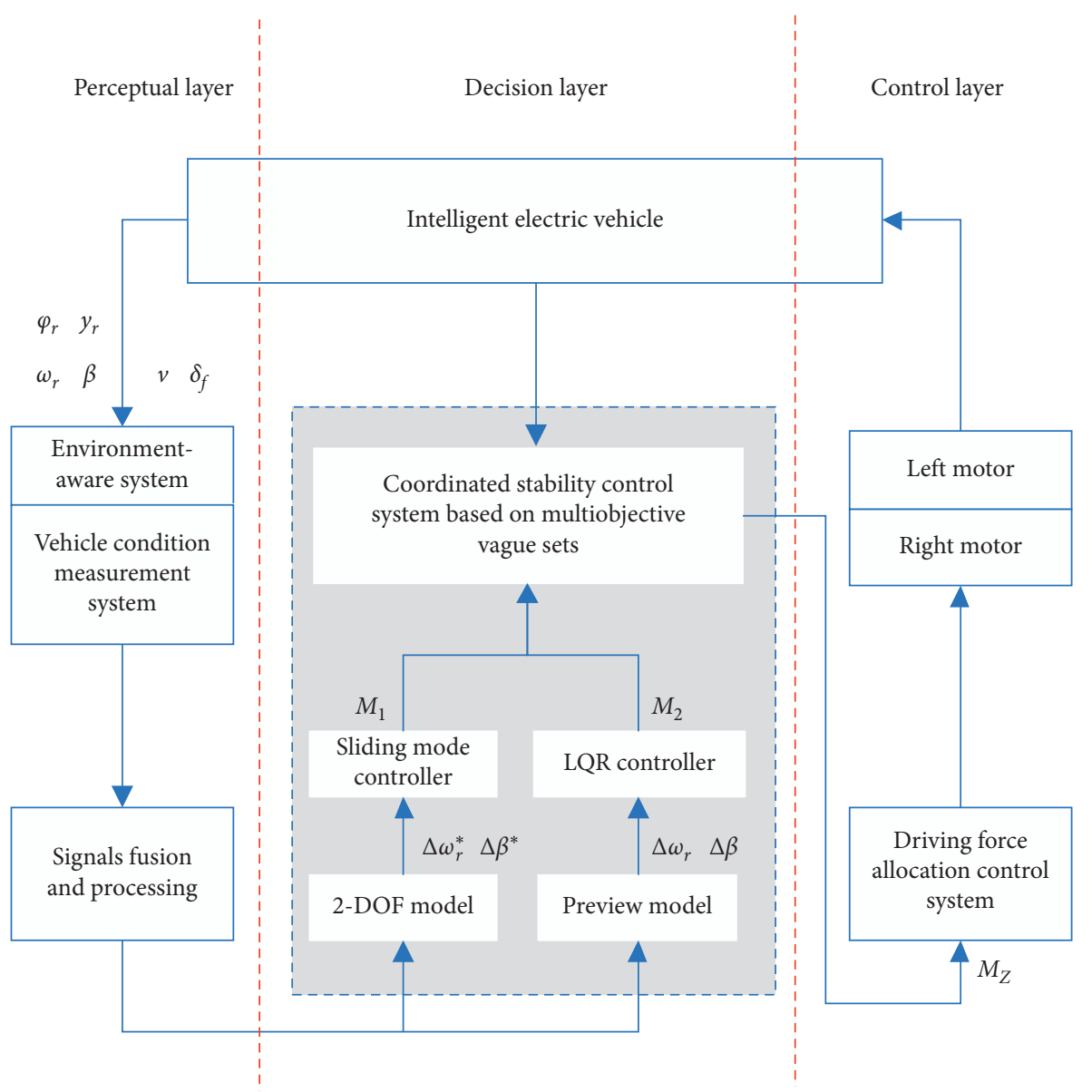

FIGURE 7: Structure of the proposed coordinated stability control strategy which consists of perceptual layer, decision layer, and control layer.

According to the yaw moment obtained, the torque distribution of each driving wheel can be completed by taking the advantage of independent control for the distributed drive vehicle.

\section{Verification and Analysis of the Proposed Tracking Stability Control Strategy}

6.1. Construction of MATLAB/CarSim Simulation Platform. In order to verify the pros and cons of the coordinated control strategy based on Vague set for tracking stability performance of intelligent electric vehicle, considering the advantages of MATLAB and CarSim software, the tracking stability control strategy model is built with MATLAB/ CarSim simulation environment.

The vehicle model is built with CarSim and the stability control strategy model is built based on MATLAB. The sideslip angle and yaw rate are obtained from the vehicle, and the deviations of sideslip angle and yaw rate are calculated with the ideal model. Then the direct yaw moments under the two control objectives are designed. According to Vague set theory, the weight factors of coordinated control are obtained, which can be used to determine the final stability control moment, so as to adjust the vehicle stability state in real time, and a complete closed loop control system is formed in this way. Two parts of the model are calculated by solvers in different software platforms, and data interact through the interface module of CarSim software. The structure diagram of the MATLAB/CarSim simulation platform for the coordinated stability control strategy of the intelligent electric vehicle is shown in Figure 8.

The premise of implementing MATLAB/CarSim simulation is to configure the input and the output parameter interface correctly. The parameters of CarSim input and output interfaces are shown in Table 1.

6.2. Simulation Results and Analyses. Based on the simulation platform of Figure 8, the comparison and analysis of simulation for the stability control strategies are carried out under the condition of double lane change, which is a typical condition for vehicle stability test.

During the simulation, the vehicle speed is maintained at $80 \mathrm{~km} / \mathrm{h}$ and road friction coefficient is set to 0.8 . The main parameters for vehicle model are chosen as Table 2. Besides, for SMC controller, $\gamma$ is set to $0.5, k_{\mathrm{SMC}}$ is set to 2000 [43], and for LQR controller, $q_{1}$ and $q_{2}$ are both set to $5 ; \mathbf{R}_{2}$ is chosen as 1 for computational convenience.

Vehicle states without stability control strategy and with traditional stability control strategy as well as the 


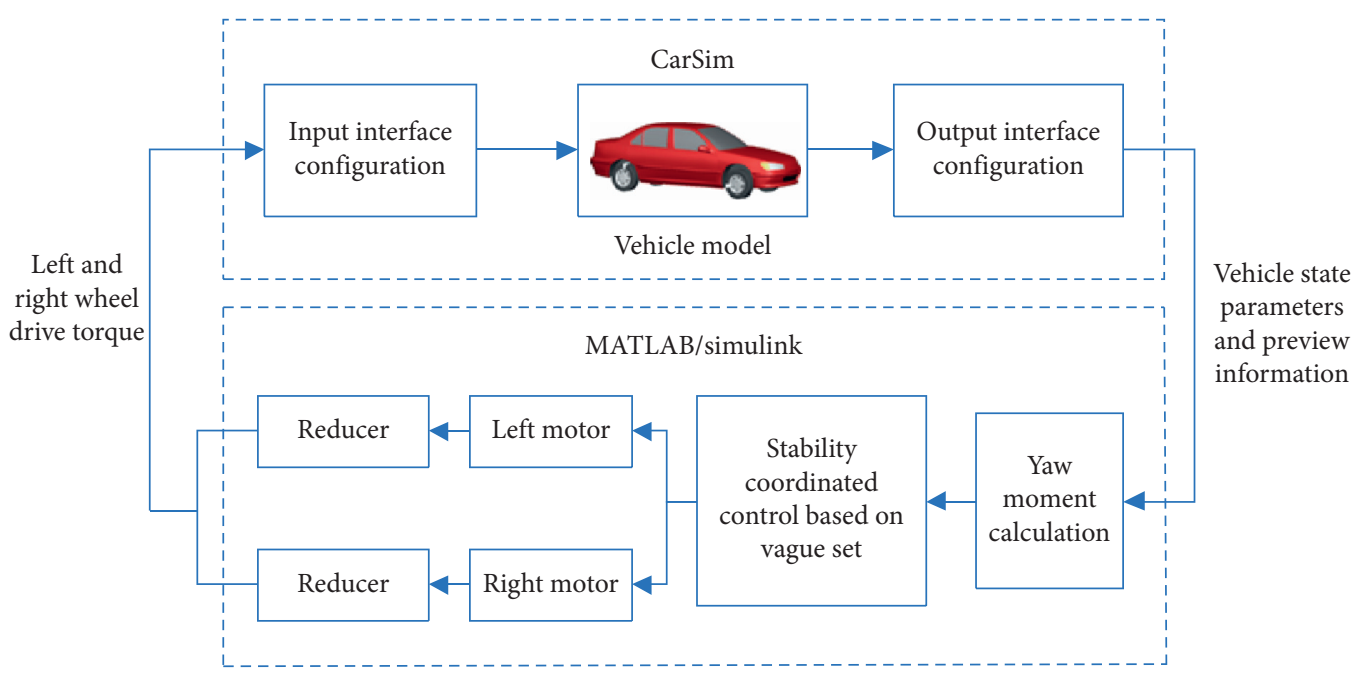

FIGURE 8: Structure diagram of MATLAB/CarSim simulation platform. CarSim and MATLAB interact with each other through input and output interfaces. Vehicle state and preview information transfer to MATLAB to calculate the desired motor torques and the torques act on the vehicle in CarSim.

TABle 1: Parameters of CarSim input and output interfaces.

\begin{tabular}{lcc}
\hline Type & Items & Parameters \\
\hline & IMP_MY_OUT_D1_L & Left front wheel torque \\
Right front wheel torque \\
Preview distance
\end{tabular}

TABLE 2: Main parameters of the vehicle model.

\begin{tabular}{lc}
\hline Items & Value \\
\hline Vehicle mass (kg) & 840 \\
Sprung mass (kg) & 586 \\
Distance of C.G. from the front axle (m) & 1.21 \\
Distance of C.G. from the rear axle (m) & 0.86 \\
Vehicle tread (m) & 1.481 \\
Effective radius of wheels (m) & 0.272 \\
Tire model & $165 /$ \\
& $65 \mathrm{R} 13$ \\
Range of steering wheel angle $\left({ }^{\circ}\right)$ & $-540 \sim 540$ \\
Ratio of driving system & 18 \\
Equivalent nominal front tire cornering stiffness (N/ & -49000 \\
rad) & \\
Equivalent nominal rear tire cornering stiffness (N/ & -50160 \\
rad) & 1523 \\
Yaw moment of inertia of vehicle $\left(\mathrm{kg} \cdot \mathrm{m}^{2}\right)$ & 2 \\
Driving motor number &
\end{tabular}

coordinated stability control strategy proposed in this paper are compared. Besides, for better comparison, the MPC control strategy is also rebuilt [50]. The yaw rate, sideslip angle, lateral acceleration, steering wheel angle, and trajectory of the vehicle are shown in Figure 9.

From Figure 9(a), it can be seen that when the intelligent electric vehicle begins to change lane, compared with the traditional stability control, MPC stability control, and coordinate stability control, yaw rate fluctuation range is larger if there is not any stability control act, and the range of yaw rate even reaches $-17.4^{\circ} / \mathrm{s} \sim 23.40^{\circ} \mathrm{s}$. While the yaw rate can be controlled within a certain range by stability control strategies, the range is $-14.36^{\circ} / \mathrm{s} \sim 13.63^{\circ} / \mathrm{s},-14.98^{\circ} / \mathrm{s} \sim 14.25^{\circ} /$ $\mathrm{s}$, and $-12.72 \% \sim 12.50 \%$ with traditional control strategy, MPC, and coordinated stability control strategy, respectively. The peak yaw rate is reduced by $46.6 \%, 11.4 \%$, and $15.0 \%$ compared with the uncontrolled situation, traditional control situation, and MPC strategy.

It can be seen from Figure 9(b) that the sideslip angle starts to fluctuate in a large range without stability control when changing lane. The range is as wide as $-3.31^{\circ} \sim 3.40^{\circ}$ during the whole process, and the vehicle is inclined to become unstable. With the traditional stability control strategy and the MPC strategy, the sideslip angle range is $-2.42^{\circ} \sim 1.94^{\circ}$ and $-2.22^{\circ} \sim 2.06^{\circ}$. And with the proposed 

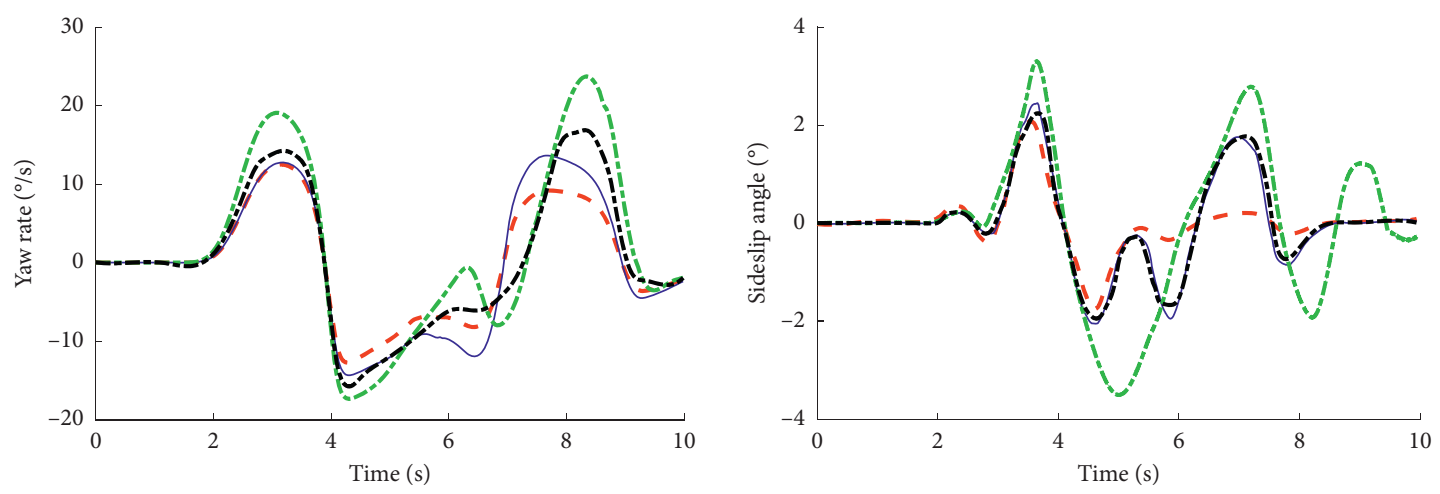

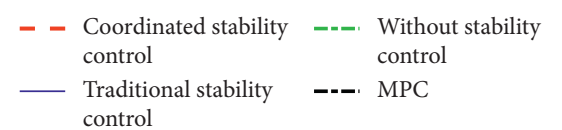

(a)

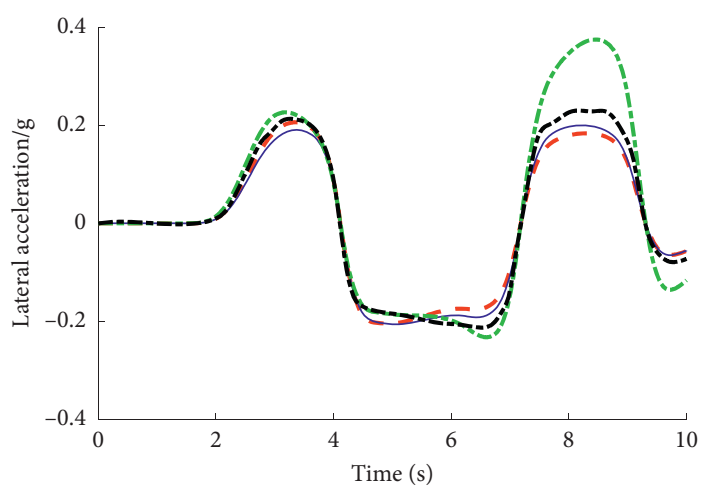

$\begin{array}{lll}\text { - } \begin{array}{l}\text { Coordinated stability } \\ \text { control }\end{array} & --- & \text { Without stability } \\ \text { Traditional stability } & \text {---. } & \text { MPC }\end{array}$

control (c)

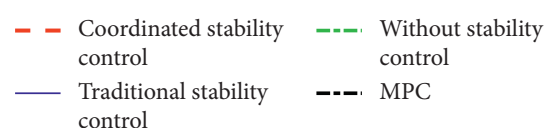

(b)

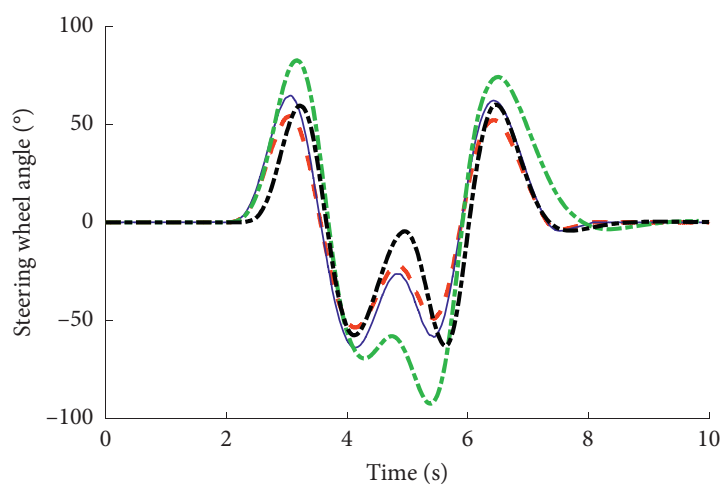

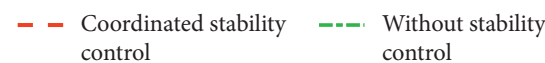

— Traditional stability _---. $\mathrm{MPC}$

(d)

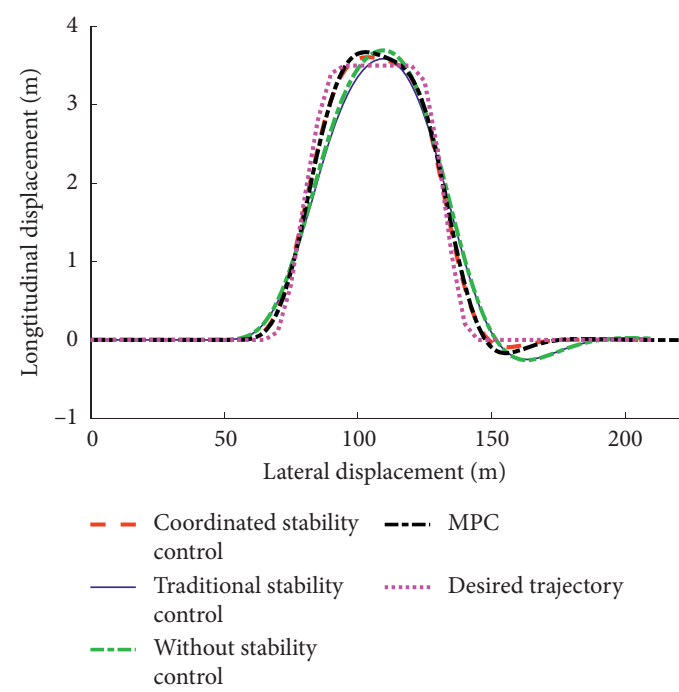

(e)

Figure 9: Simulation results of double lane change under various control strategies. (a) Yaw rate. (b) Sideslip angle. (c) Lateral acceleration. (d) Steering angle. (e) Trajectories. 


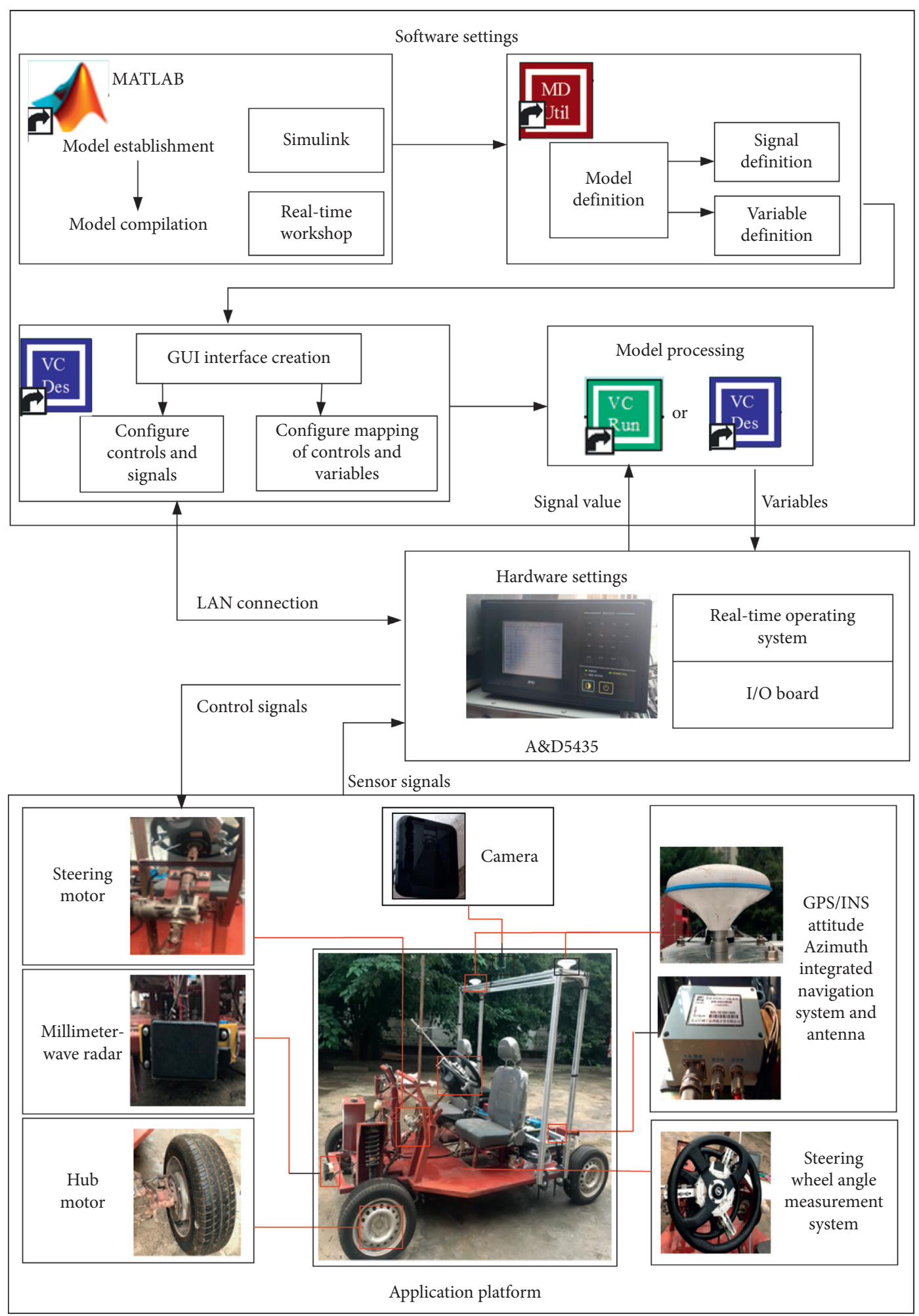

FIGURE 10: Intelligent electric vehicle testing platform, which consists of software settings, hardware settings, and an application platform.

coordinated control strategy, the range is only $-2.10^{\circ} \sim 1.83^{\circ}$. The peak value is reduced by $45.6 \%, 13.2 \%$, and $11.2 \%$ compared with the uncontrolled situation and with the traditional stability control as well as MPC strategy. Vehicle sideslip angle is significantly reduced.
From the curve of lateral acceleration in Figure 9(c), it can be seen that, without stability control strategy, the lateral acceleration increases significantly and the peak value reaches $0.375 \mathrm{~g}$ during the second lane change stage. The peak value of lateral acceleration is $0.201 \mathrm{~g}$ and $0.219 \mathrm{~g}$ with 


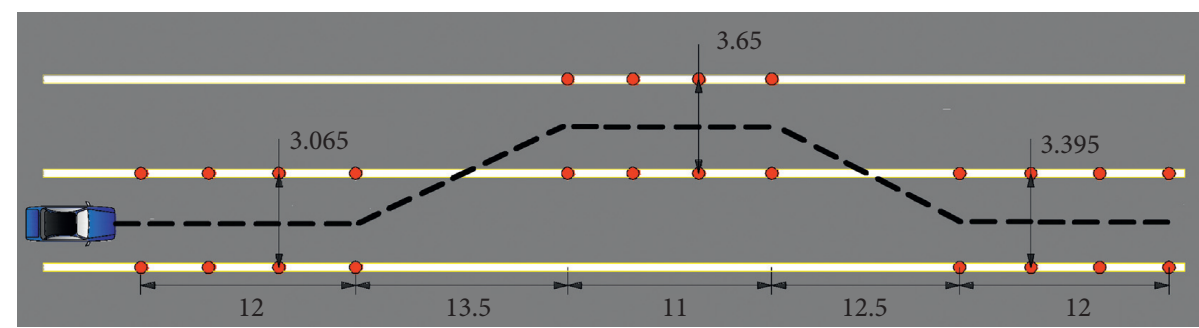

Figure 11: Double lane change setting, which shows the geometric dimensions and position relationships of the tested lanes.

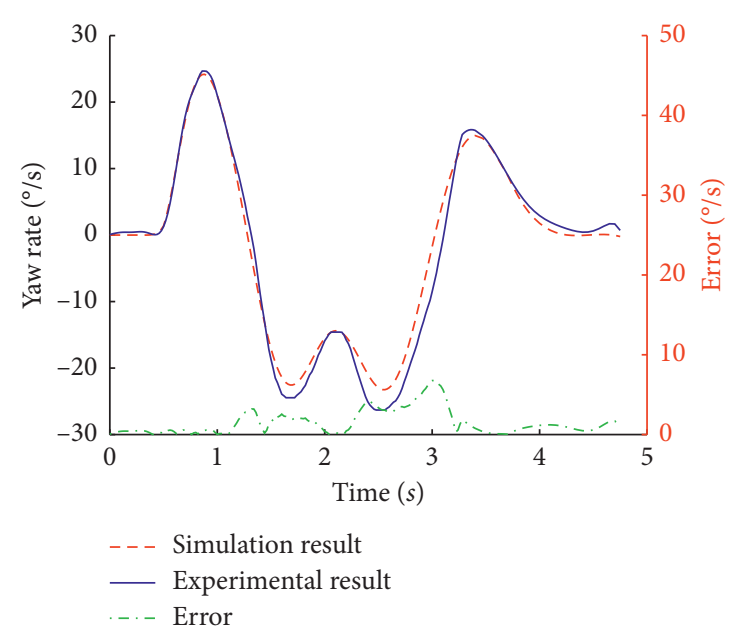

(a)

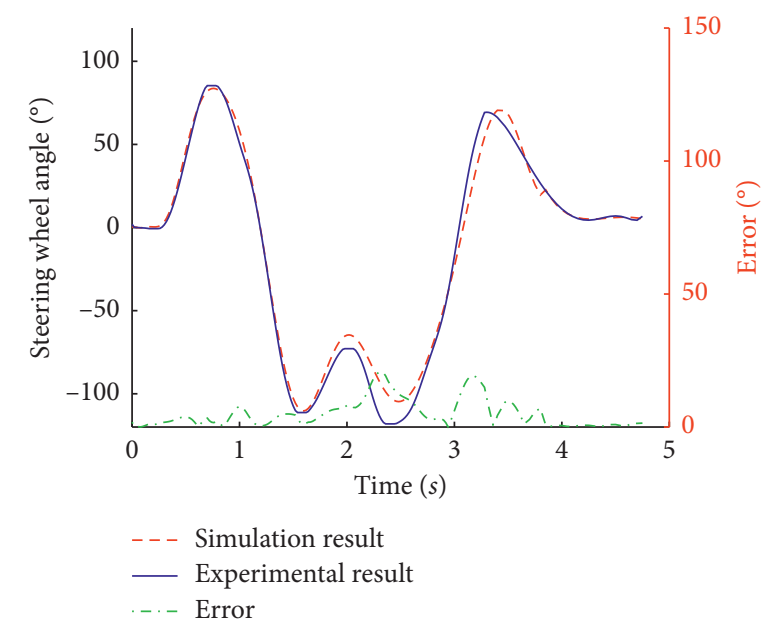

(c)

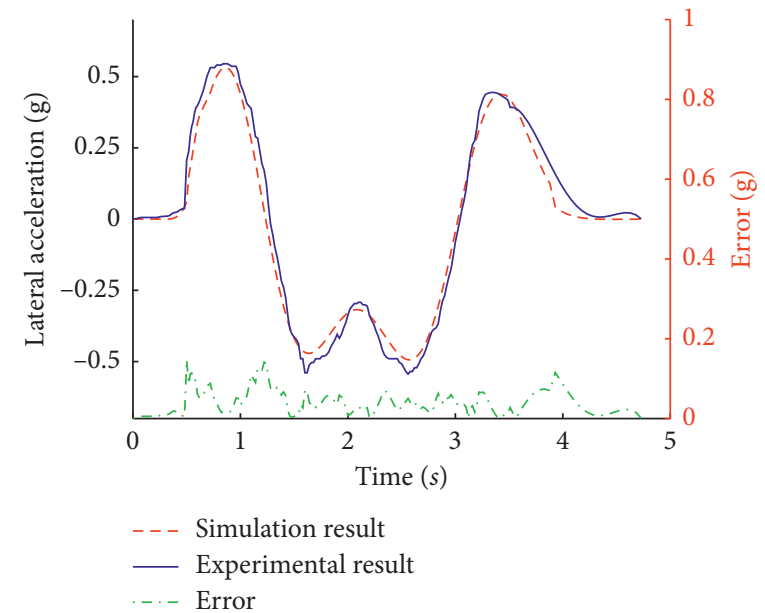

(b)

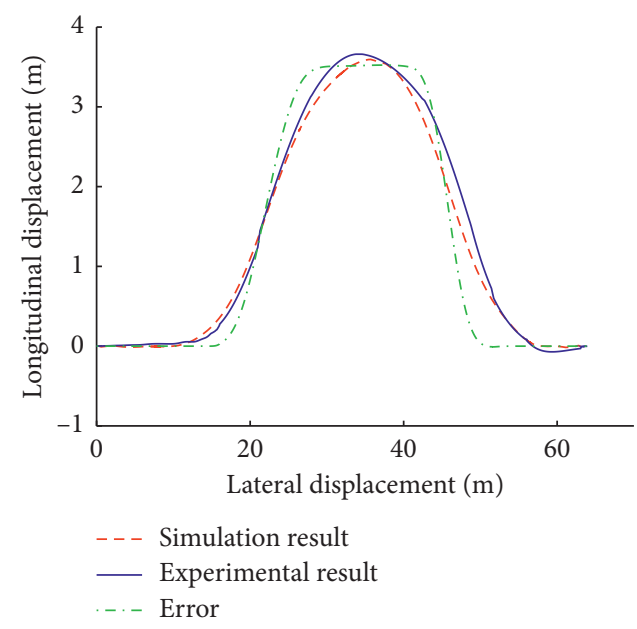

(d)

Figure 12: Experiments at the speed of $45 \mathrm{~km} / \mathrm{h}$. (a) Yaw rate. (b) Lateral acceleration. (c) Steering wheel angle. (d) Trajectories.

traditional stability control strategy and MPC strategy, while that peak value is only $0.183 \mathrm{~g}$ with the proposed coordinated stability control strategy. The value is $51.2 \%, 8.90 \%$, and $16.4 \%$ lower than that without any stability control and with traditional stability control and MPC control.

From Figure 9(d), it can be seen that steering wheel angle fluctuates in the range of $-92.53^{\circ} \sim 82.62^{\circ}$ without stability control strategy during the lane changing process. When the stability control strategy is applied, the range decreases. With traditional dynamic stability control strategy and MPC strategy, the range is $-63.86^{\circ} \sim 64.55^{\circ}$ and $-62.53^{\circ} \sim 59.53^{\circ}$, and with the stability control strategy proposed in this paper, the range is only $-53.64^{\circ} \sim 54.18^{\circ}$.

As can be seen from Figure 9(e), the trajectories of the intelligent electric vehicle can follow the desired trend without control strategy. Although the deviation between them is accepted, a smaller deviation can be obtained with the coordinated stability control strategy proposed in this paper.

In summary, aiming at tracking stability problem, the strategy developed in this paper has good performance 


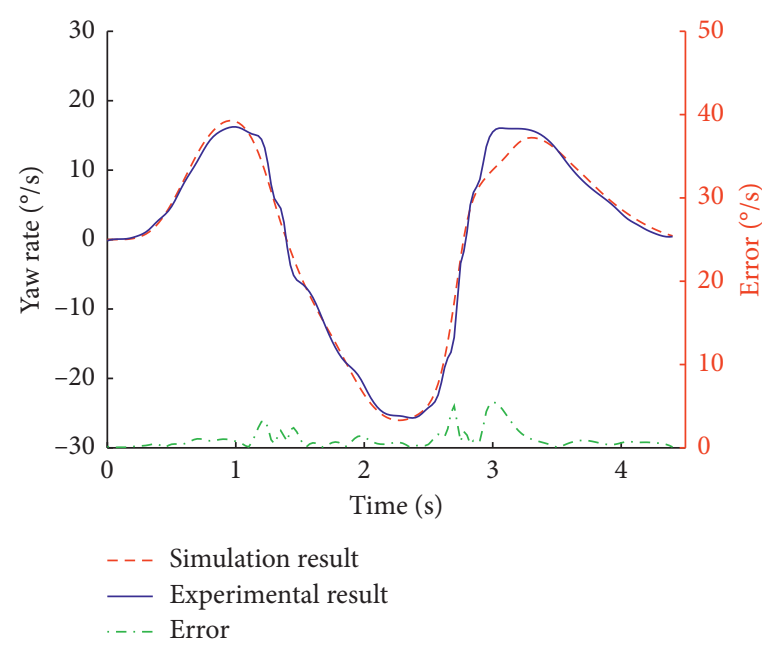

(a)

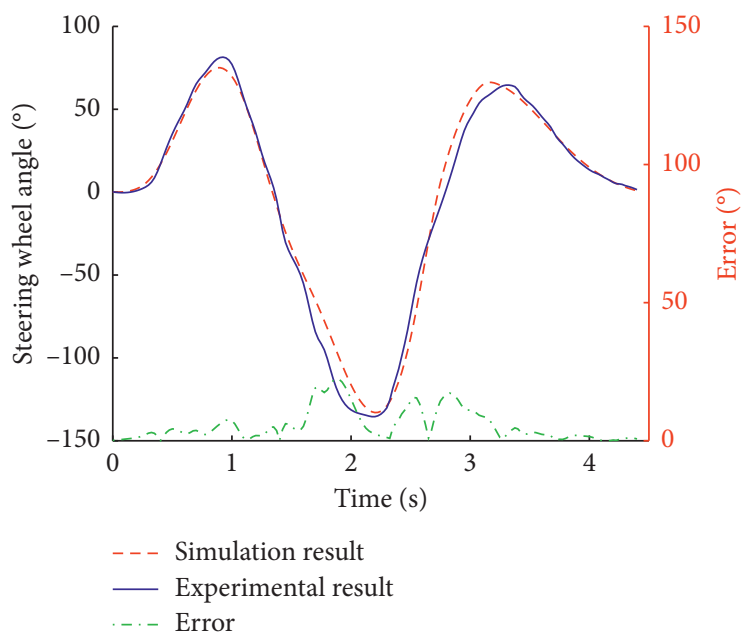

(c)

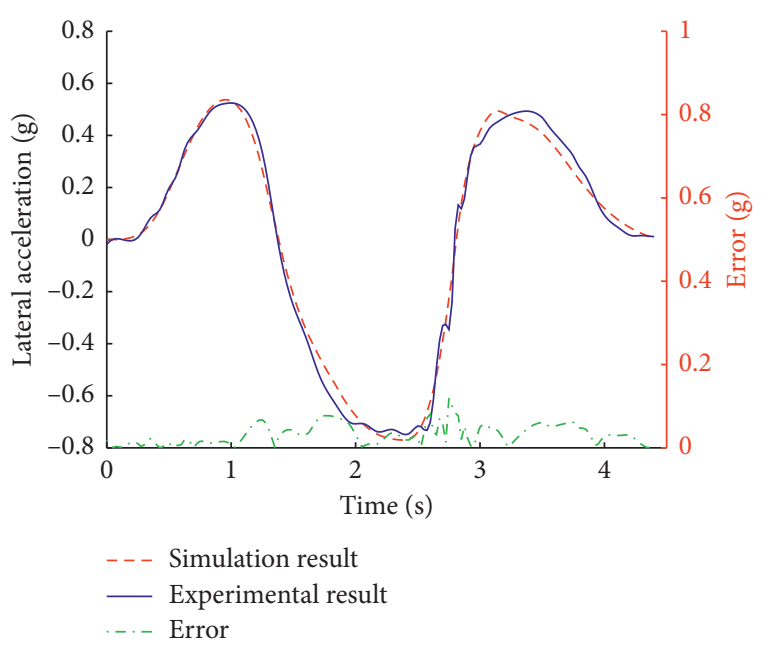

(b)

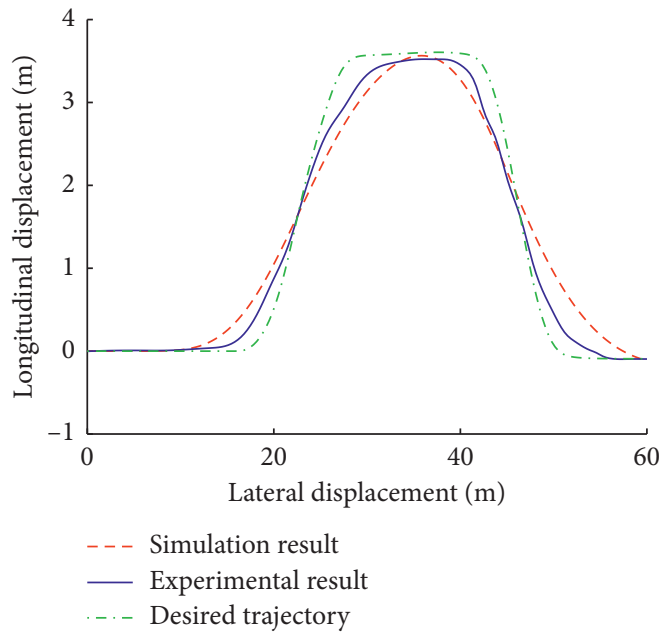

(d)

Figure 13: Experiments at the speed of $60 \mathrm{~km} / \mathrm{h}$. (a) Yaw rate. (b) Lateral acceleration. (c) Steering wheel angle. (d) Trajectories.

compared with the stability control strategies failing to take preview into consideration. Vehicle states including yaw rate, sideslip angle, lateral acceleration, and steering wheel angle are limited in an acceptable range. What is more, the proposed strategy is also beneficial to tracking accuracy rising.

6.3. Vehicle Test Verifications and Analyses. To further verify the proposed control strategy, a hardware-in-loop experiment platform for front-wheel independent drive intelligent electric vehicle is built with A\&D5435, as shown in Figure 10. The simulation model is converted into $\mathrm{C}$ code by real-time workshop technology so that the control strategy can be downloaded to A\&D5435, where the control algorithm runs. The information of vehicle position, vehicle speed, yaw angle, and yaw rate is collected by GPS/INS. Steering wheel angle and its changing rate are collected by steering wheel angle sensor. These states are transmitted through CAN bus to A\&D5435, and the PWM control signal is produced to control the driving motor according to vehicle state. At the same time, A\&D5435 can simultaneously display, record, and save the state information of intelligent electric vehicle during the test. The parameters of the test vehicle are similar to the vehicle model in simulation.

In this paper, the test of double lane change with constant speed is carried out. The lane is shown in Figure 11. The red dots are cones forming the boundary of lanes. Vehicle yaw rate, lateral acceleration, steering wheel angle, and trajectories with the speed of $45 \mathrm{~km} / \mathrm{h}$ and $60 \mathrm{~km} / \mathrm{h}$ are shown in Figures 12 and 13, respectively.

Vehicle yaw rate is plotted in Figure 12(a). The range of the experimental value and simulation value is $-26.31 \%$ to $24.66^{\circ}$ s and $-23.65 \%$ to $24.43 \%$, respectively. The maximum deviation is $2.42 \%$, which occurs at the second lane changing point. The average deviation between them is only $1.22^{\circ}$ s. Yaw rate can be controlled within a certain range through the control strategy researched in this paper.

The lateral acceleration of the vehicle is shown in Figure 12(b). The value approximately maintains zero along the straight line. With the change of yaw rate, the lateral acceleration also changes. In the driving course, the 
acceleration range obtained in the experiment is $-0.533 \mathrm{~g}$ to $0.545 \mathrm{~g}$ and is $-0.492 \mathrm{~g}$ to $0.538 \mathrm{~g}$ in the simulation. The maximum deviation and average deviation between them are $0.04 \mathrm{~g}$ and $0.01 \mathrm{~g}$, respectively, and their variation tendency is the same, which shows good consistency in experiment and simulation.

From Figure 12(c), it can be seen that the steering wheel angle changes smoothly during the whole driving process without large fluctuation, which is beneficial for the vehicle stability. The value fluctuates from $-108.76^{\circ}$ to $78.02^{\circ}$ in experiment and $-112.43^{\circ}$ to $75.47^{\circ}$ in simulation. The error between them is within $8.60^{\circ}$ during the whole process, which shows that the experimental results are in good agreement with the simulation results.

Vehicle trajectories are plotted in Figure 12(d). It can be seen that the vehicle can travel along the given trajectory in both experiment and simulation, and the experimental result is close to the simulation result. Besides, deviations between desired trajectory and the actual ones are also accepted; it is obvious that the stability control strategy is also beneficial to improving the tracking performance.

From Figures 13(a)-13(c), it can be seen that the fluctuation tendency of yaw rate, lateral acceleration, and steering wheel angle of the vehicle with speed of $60 \mathrm{~km} / \mathrm{h}$ is similar to that of $45 \mathrm{~km} / \mathrm{h}$, and experimental results are also consistent with simulation results. At $2.32 \mathrm{~s}$, the amplitude of steering is the largest, and the maximum values of test results and simulation results are $-135.40^{\circ}$ and $-132.71^{\circ}$, respectively, with an average error of $7.73^{\circ}$. The range of yaw rate obtained from experiment and simulation varies from $-25.71 \%$ s to $16.05 \%$ and $-26.02 \%$ s to $17.12 \%$, with an average error of $5.65 \%$ s. And for lateral acceleration, the range varies from $-0.736 \mathrm{~g}$ to $0.516 \mathrm{~g}$ and $-0.756 \mathrm{~g}$ to $0.535 \mathrm{~g}$, with an average error of $0.03 \mathrm{~g}$. The maximum errors between simulation results and experimental results for yaw rate, lateral acceleration, and steering wheel angle are restrained within $5.43^{\circ} / \mathrm{s}, 0.12 \mathrm{~g}$, and $21.0^{\circ}$, respectively. From Figure $13(\mathrm{~d})$, it can be seen that the vehicle trajectory variation of experimental result and simulation result is similar. Before lane changing, the trajectory deviation between them is quite small, and there is a certain deviation when steering. As a whole, the stability control strategy can not only improve the tracking stability performance but also have a positive effect on tracking precision.

\section{Conclusions}

This paper proposes a novel tracking stability control strategy based on traditional stability control with adoption of preview characteristics for intelligent electric vehicles. Traditional dynamic stability control enables the vehicle to track desired stable states; meanwhile preview model reflecting driving behavior further modifies the former control. Vague set is used to calculate the weight coefficient of the system in different driving states to ensure the outstanding trajectory tracking stability performance of the vehicle. Simulation results show that, with the proposed control strategy, the peak values of sideslip angle, yaw rate, and lateral acceleration are reduced by $13.2 \%, 11.4 \%$, and
8.9\% compared with the traditional stability control strategy. What is more, trajectories under different control strategies indicate that the proposed coordinated stability control strategy is also beneficial to improving trajectory tracking precision. Besides, a rapid prototyping experiment platform of the intelligent electric vehicle is built based on A\&D5435, with which a double lane change experiment is conducted. The average deviations between experimental result and simulation result of yaw rate, lateral acceleration, and steering wheel angle are less than $10 \%$. Simulation and experimental results demonstrate that the proposed control strategy can be applied to improve the tracking stability.

To further enhance the control accuracy and practicability, the coupling research of longitudinal dynamics and lateral dynamics will be carried out. In addition, the approach will also be extended to handle more complex and realistic traffic scenarios.

\section{Data Availability}

The data used to support the findings of this study are available from the corresponding author upon request.

\section{Conflicts of Interest}

The authors declare no conflicts of interest.

\section{Acknowledgments}

This research was funded by the National Key R\&D Program of China (2018YFB1600701), Shaanxi Province Industrial Innovation Chain Project (2018ZDCXL-GY-05-03-01), and the Youth Innovation Team of Shaanxi Universities.

\section{References}

[1] X. Zhang and X. Zhu, "Autonomous path tracking control of intelligent electric vehicles based on lane detection and optimal preview method," Expert Systems with Applications, vol. 121, pp. 38-48, 2019.

[2] J. Guo, Y. Luo, K. Li, and Y. Dai, "Coordinated path-following and direct yaw-moment control of autonomous electric vehicles with sideslip angle estimation," Mechanical Systems and Signal Processing, vol. 105, pp. 183-199, 2018.

[3] X. Li, Z. Sun, D. Cao, D. Liu, and H. He, "Development of a new integrated local trajectory planning and tracking control framework for autonomous ground vehicles," Mechanical Systems and Signal Processing, vol. 87, pp. 118-137, 2017.

[4] X. Zhao, S. Wang, J. Ma, Q. Yu, Q. Gao, and M. Yu, "Identification of driver's braking intention based on a hybrid model of GHMM and GGAP-RBFNN," Neural Computing and Applications, vol. 31, no. S1, pp. 161-174, 2018.

[5] M. Brown, J. Funke, S. Erlien, and J. C. Gerdes, "Safe driving envelopes for path tracking in autonomous vehicles," Control Engineering Practice, vol. 61, pp. 307-316, 2017.

[6] X. Huang, "Modeling and simulation of robust control law of electric power steering system in intelligent electric vehicle," Computers and Security, vol. 30, pp. 176-180, 2013.

[7] C. Yang, Z. Li, and J. Li, "Trajectory planning and optimized adaptive control for a class of wheeled inverted pendulum vehicle models," IEEE Transaction on Cybernetics, vol. 43, pp. 24-36, 2013. 
[8] M. Silva, L. Garrote, F. Moita et al., "Autonomous electric vehicle: steering and path-following control systems," in Proceedings of the 2012 16th IEEE Mediterranean Electrotechnical Conference, pp. 442-445, Yasmine Hammamet, Tunisia, March 2012.

[9] G. Bartolini, A. Pisano, E. Punta, and E. Usai, "Simplex sliding mode control for autonomous six-DOF vehicles with monodirectional actuators: robustness, stability, and implementation issues," International Journal of Robust and Nonlinear Control, vol. 29, no. 3, pp. 529-549, 2019.

[10] K. Nam, H. Fujimoto, and Y. Hori, "Design of an adaptive sliding mode controller for robust yaw stabilization of in-wheel-motor-driven electric vehicles," International Journal of Vehicle Designing, vol. 67, pp. 98-113, 2014.

[11] J. D. J. Rubio, "Robust feedback linearization for nonlinear processes control," ISA Transactions, vol. 74, pp. 155-164, 2018.

[12] J. D. J. Rubio, J. F. Novoa, G. Ochoa et al., "Structure regulator for the perturbations attenuation in a quadrotor," IEEE Access, vol. 7, no. 1, pp. 138244-138252, 2019.

[13] J. Kumar, V. Kumar, and K. P. S. Rana, "Design of robust fractional order fuzzy sliding mode PID controller for two link robotic manipulator system," Journal of Intelligent \& Fuzzy Systems, vol. 35, no. 5, pp. 5301-5315, 2018.

[14] A. Arturo, J. A. Meda-Campaña, G. Ochoa et al., "An electricity generator based on the interaction of static and dynamic magnets," IEEE Transactions on Magnetics, vol. 55, no. 8, Article ID 8204511, 2019.

[15] Y. Hu, W. Gu, H. Zhang, and H. Chen, "Adaptive robust triple-step control for compensating cogging torque and model uncertainty in a DC motor," IEEE Transactions on System, Man, and Cybernetics: System, vol. 49, no. 12, pp. 1396-2405, 2019.

[16] Z. Guan, J. Ding, and F. Du, "Simulation research on trajectory tracking controller based on MPC algorithm," in Proceedings of the 2017 2nd International Conference on Robotics and Automation Engineering (ICRAE), pp. 212-216, Shanghai, China, Deceeber 2017.

[17] J. Ji, A. Khajepour, W. W. Melek, and Y. Huang, "Path planning and tracking for vehicle collision avoidance based on model predictive control with multiconstraints," IEEE Transactions on Vehicular Technology, vol. 66, no. 2, pp. 952-964, 2017.

[18] H. Guo, D. Cao, H. Chen, Z. Sun, and Y. Hu, "Model predictive path following control for autonomous cars considering a measurable disturbance: implementation, testing, and verification," Mechanical Systems and Signal Processing, vol. 118, pp. 41-60, 2019.

[19] Y. Yoon, J. Shin, H. J. Kim, Y. Park, and S. Sastry, "Modelpredictive active steering and obstacle avoidance for autonomous ground vehicles," Control Engineering Practice, vol. 17, no. 7, pp. 741-750, 2009.

[20] P. Song, C. Zong, and M. Tomizuka, "Combined longitudinal and lateral control for automated lane guidance of full driveby-wire vehicles," SAE International Journal of Passenger Cars-Electronic and Electrical Systems, vol. 8, no. 2, pp. 419-424, 2015.

[21] L.-Y. Hsu and T.-L. Chen, "An optimal wheel torque distribution controller for automated vehicle trajectory following," IEEE Transactions on Vehicular Technology, vol. 62, no. 6, pp. 2430-2440, 2013.

[22] P. Raksincharoensak, M. Nagai, and M. Shino, "Lane keeping control strategy with direct yaw moment control input by considering dynamics of electric vehicle," Vehicle System Dynamics, vol. 44, no. 1, pp. 192-201, 2006.

[23] G. V. Raffo, G. K. Gomes, J. E. Normey-Rico, C. R. Kelber, and L. B. Becker, "A predictive controller for autonomous vehicle path tracking," IEEE Transactions on Intelligent Transportation Systems, vol. 10, no. 1, pp. 92-102, 2009.

[24] A. Goodarzi and M. Mohammadi, "Stability enhancement and fuel economy of the 4-wheel-drive hybrid electric vehicles by optimal tyre force distribution," Vehicle System Dynamics, vol. 52, no. 4, pp. 539-561, 2014.

[25] H. Zheng and S. Yang, "A trajectory tracking control strategy of 4WIS/4WID electric vehicle with adaptation of driving conditions," Applied Sciences, vol. 9, no. 1, p. 168, 2019.

[26] L. Yang, M. Yue, and T. Ma, "Path following predictive control for autonomous vehicles subject to uncertain tireground adhesion and varied road curvature," International Journal of Control, Automation and Systems, vol. 17, no. 1, pp. 193-202, 2019.

[27] F. Lin, Y. W. Zhang, Y. Q. Zhao et al., "Trajectory tracking of autonomous vehicle with the fusion of DYC and longitudinallateral control," Chinese Journal of Mechanical Engineering, vol. 32, p. 16, 2019.

[28] G. Zou, Y. Liu, and K. Li, “Tire longitudinal force optimization distribution for independent 4WD EV," Journal of Tsinghua University (Philosophy and Social Sciences), vol. 49, pp. 719$722,2009$.

[29] X. Zhao, Q. Yu, M. Yu et al., "Research on an equal power allocation electronic differential system for electric vehicle with dual-wheeled-motor front drive based on a wavelet controller," Advances in Mechanical Engineering, vol. 10, pp. 1-24, 2018.

[30] Y. L. He, J. Ma, X. Zhao et al., "Research on wavelet control based stability of the electric vehicle," Chinese Journal of Highway and Transportation, vol. 31, pp. 214-226, 2018.

[31] V. Ivanov, D. Savitski, K. Augsburg, P. Barber, B. Knauder, and J. Zehetner, "Wheel slip control for all-wheel drive electric vehicle with compensation of road disturbances," Journal of Terramechanics, vol. 61, pp. 1-10, 2015.

[32] X. Zhao, Q. Yu, J. Ma, Y. Wu, M. Yu, and Y. Ye, "Development of a representative EV urban driving cycle based on a k-means and SVM hybrid clustering algorithm," Journal of Advanced Transportation, vol. 2018, Article ID 1890753, 18 pages, 2018.

[33] J. Ryan, J. Lu, and D. Bevly, "State estimation for vehicle stability control: a kinematic approach using only GPS and VSC sensors," in Proceedings of the ASME Dynamic Systems and Control Conference, pp. 773-780, Cambridge, MA, USA, September 2010.

[34] X. Zhang, K. Wei, X. Yuan et al., "Optimal torque distribution for the stability improvement of a four-wheel distributeddriven electric vehicle using coordinated control," Journal of Computational and Nonlinear Dynamics, vol. 11, no. 5, Article ID 051017, 2016.

[35] F. Yu and Y. Lin, "Automobile System Dynamics," China Machine Press, Beijing, China, 2005.

[36] H. Pacejka, "Tire and Vehicle Dynamics," Elsevier, Oxford, UK, 3rd edition, 2012.

[37] H. Pacejka and I. Besselink, "Magic formula tyre model with transient properties," Vehicle System of Dynamics, vol. 27, no. 1, pp. 234-249, 1997.

[38] L. Li, G. Jia, J. Chen, H. Zhu, D. Cao, and J. Song, "A novel vehicle dynamics stability control algorithm based on the hierarchical strategy with constrain of nonlinear tyre forces," Vehicle System Dynamics, vol. 53, no. 8, pp. 1093-1116, 2015. 
[39] J. Guo, Y. Luo, C. Hu, C. Tao, and K. Li, "Robust combined lane keeping and direct yaw moment control for intelligent electric vehicles with time delay," International Journal of Automotive Technology, vol. 20, no. 2, pp. 289-296, 2019.

[40] A. Matsushima and S. Tsugawa, "Look-ahead-distance for lateral control of vision-based vehicles," in Proceedings of the 17th ITS World Congress, pp. 25-29, Busan, South Korea, October 2010.

[41] Z. Wang, Y. Wang, L. Zhang, and M. Liu, "Vehicle stability enhancement through hierarchical control for a four-wheelindependently-actuated electric vehicle," Energies, vol. 10, no. 7, p. 947, 2017.

[42] R. Hou, L. Zhai, T. Sun, Y. Hou, and G. Hu, "Steering stability control of a four in-wheel motor drive electric vehicle on a road with varying adhesion coefficient," IEEE Access, vol. 7, pp. 32617-32627, 2019.

[43] S. Ding, L. Liu, and W. Zheng, "Sliding mode direct yawmoment control design for in-wheel electric vehicles," IEEE Transactions on Industrial Electronics, vol. 64, no. 8, pp. 6752-6762, 2017.

[44] S. Li, Q. Li, H. Liu et al., "Sliding mode variable structure strategy for vehicle stability control," Journal of Jilin University (Engineering and Technology), vol. 49, pp. 1288-1292, 2019.

[45] P. Raksincharoensak, T. Mizushima, and M. Nagai, "Direct yaw moment control system based on driver behaviour recognition," Vehicle System Dynamics, vol. 46, no. 1, pp. 911-921, 2008.

[46] W. Jiang and H. Huang, "Analysis and simulation for vehicle stability control system based on a spatial previewed driver model," Automobile Technology, vol. 12, pp. 27-31, 2011.

[47] T. Lee, J. Kang, K. Yi, and K. Noh, "An investigation on the integrated human driver model for closed-loop simulation of intelligent safety systems," Journal of Mechanical Science and Technology, vol. 24, no. 3, pp. 761-767, 2010.

[48] H. Ding, K. Guo, and H. Chen, "LQR method for vehicle yaw moment decision in vehicle stability control," Jilin University (Engineering and Technology Edition), vol. 3, pp. 597-601, 2010.

[49] J. Luo and J. Xie, "Fuzzy multi objectives decision making based on vague set and its application," Mathematics in Practice and Theory, vol. 38, pp. 114-122, 2008.

[50] B. Ren, H. Chen, H. Zhao et al., "MPC-based yaw stability control in in-wheel-motored EV via active front steering and motor torque distribution," Mechatronics, vol. 38, pp. 103114, 2016. 